\title{
COARSE FLOW SPACES FOR RELATIVELY HYPERBOLIC GROUPS
}

\author{
BARTELS, A.
}

\begin{abstract}
We introduce coarse flow spaces for relatively hyperbolic groups and use them to verify a regularity condition for the action of relatively hyperbolic groups on their boundaries. As an application the Farrell-Jones Conjecture for relatively hyperbolic groups can be reduced to the peripheral subgroups (up to index 2 overgroups in the $L$-theory case).
\end{abstract}

\section{INTRODUCTION}

Farrell and Jones [19] used the geodesic flow on closed Riemannian manifolds of negative sectional curvature to prove that the Whitehead group of the fundamental group of such manifolds vanishes. This method has been extremely fruitful and has been generalized in many ways.

Among the developments following [19] was the formulation of what is now known as the Farrell-Jones Conjecture [20]. This conjecture predicts that the $K$ - and $L$ theory of group rings $R[G]$ is determined by group homology and the $K$ - and $L$-theory of group rings of virtually cyclic subgroups. If the conjecture holds for a group $G$, then this often yields vanishing results or computational results for Whitehead groups and the manifolds structure set appearing in surgery theory. In particular, the Farrell-Jones Conjecture has implications for the classification of higher dimensional non-simply connected manifolds. We will review the precise formulation of the conjecture in Section 4. More information about the FarrellJones Conjecture and its applications can be found for example in $\mathbf{7}, 27,28$.

In many cases it is fruitful to replace the family of virtually cyclic subgroups VCyc with a bigger family of subgroups $\mathcal{F}$. There is then a formulation of the Farrell-Jones Conjecture relative to $\mathcal{F}$. This version of the conjecture is particularly useful whenever the groups in the family $\mathcal{F}$ are already known to satisfy the original Farrell-Jones Conjecture.

In work with Lück and Reich the geodesic flow method from [19] has been successfully implemented in [3, 5, 6] to prove the Farrell-Jones Conjecture for hyperbolic groups. More generally, the results from 3, 6, state that the Farrell-Jones Conjecture for a group $G$ holds relative to a family $\mathcal{F}$ whenever there exists an action of $G$ on a finite dimensional contractible $A N R$ satisfying a regularity condition relative to the family $\mathcal{F}$. We will review this regularity condition shortly and refer to actions satisfying it relative to $\mathcal{F}$ as finitely $\mathcal{F}$-amenable actions. In this language the main result of [5] implies that for hyperbolic groups the action on the boundary is finitely VCyc-amenable. In this paper we prove a similar result for relatively hyperbolic groups. For definitions of relatively hyperbolic groups see [18, 22, 23, 40. We will use Bowditch's point of view [13, recalled in Section 2.

Theorem. Let $G$ be a countable group that is relatively hyperbolic to subgroups $P_{1}, \ldots, P_{n}$. Let $\mathcal{P}$ be the family of subgroups of $G$ that are either virtually cyclic or

Date: July 2016

2010 Mathematics Subject Classification. 18F25, 19G24, $20 \mathrm{~F} 67$.

Key words and phrases. Farrell-Jones Conjecture, $K$ - and $L$-theory of group rings. 
subconjugated to one of the $P_{i}$. Then the action of $G$ on its boundary $\Delta$ is finitely $\mathcal{P}$-amenable.

This result appears as Theorem 3.1 in Section 3

The boundary $\Delta$ is usually neither contractible nor an $A N R$. For hyperbolic groups Bestvina-Mess proved that the union of the Rips complex with the boundary is a contractible compact $A N R$ [11. Similarly, for relatively hyperbolic groups there is a relative Rips complex [15, 30] and in the appendix we extend the BestvinaMess result to the relatively hyperbolic case. This is closely related to results of Dahmani [15]. Using [3, 6] we will obtain the following application to the FarrellJones Conjecture.

Corollary. Let $G$ be a countable group that is relatively hyperbolic to subgroups $P_{1}, \ldots, P_{n}$. If $P_{1}, \ldots, P_{n}$ satisfy the Farrell-Jones Conjecture then $G$ satisfies the Farrell-Jones Conjecture.

This corollary is more carefully formulated as Corollary 4.6. Such results are typical for relatively hyperbolic groups. For example if a group $G$ is relatively hyperbolic to groups of finite asymptotic dimension, then $G$ is of finite asymptotic dimension 33 .

For groups that are relatively hyperbolic to groups that satisfy the Farrell-Jones Conjecture and are in addition residually finite Antolín, Coulon and Gandini [1] give an alternative proof of the above corollary using Dehn fillings.

A regularity condition. Let $X$ be a $G$-space and $\mathcal{F}$ be a family of subgroups of $G$. An open subset $U \subseteq X$ is said to be an $\mathcal{F}$-subset if there is $F \in \mathcal{F}$ such that $g U=U$ for $g \in F$ and $g U \cap U=\emptyset$ if $g \notin F$. A cover $\mathcal{U}$ of open subsets of $X$ is said to be $G$-invariant if $g U \in \mathcal{U}$ for all $g \in G, U \in \mathcal{U}$. A $G$-invariant cover $\mathcal{U}$ of $X$ is said to be an $\mathcal{F}$-cover if the members of $\mathcal{U}$ are all $\mathcal{F}$-subsets. The order of a collection $\mathcal{U}$ of subsets of $X$ is $\leq N$ if each $x \in X$ is contained in at most $N+1$-members of $\mathcal{U}$. If $\mathcal{U}$ is a cover, then we will often call the order of $\mathcal{U}$ the dimension of $\mathcal{U}$.

Definition 0.1. Let $G$ be a group and $\mathcal{F}$ be a family of subgroups. An action of $G$ on a space $X$ is said to be $N$ - $\mathcal{F}$-amenable if for any finite subset $S$ of $G$ there exists an open $\mathcal{F}$-cover $\mathcal{U}$ of $G \times X$ (equipped with the diagonal $G$-action) with the following properties:

a) the dimension of $\mathcal{U}$ is at most $N$;

b) for all $x \in X$ there is $U \in \mathcal{U}$ with $S \times\{x\} \subseteq U$.

An action that is $N-\mathcal{F}$-amenable for some $N$ is said to be finitely $\mathcal{F}$-amenable.

In [6] such cover were called wide.

Remark 0.2. Suppose that the action $G \curvearrowright X$ is $N$ - $\mathcal{F}$-amenable. Then all finitely generated subgroups $H$ of $G$ that fix a point $x \in X$ belong to $\mathcal{F}$. Indeed, if $S$ is symmetric, contains $e$, generates $H$ and satisfies $S \times\{x\} \subseteq U$ then $(e, x) \in s U$ for all $s \in S$ and therefore $U \cap s U \neq \emptyset$ for all $s \in S$.

Remark 0.3. Suppose that the action $G \curvearrowright X$ is such that there exists an $\mathcal{F}$ cover $\mathcal{V}$ for $X$ of dimension $N$. Then the action is $N$ - $\mathcal{F}$-amenable. Indeed, if we set $\mathcal{U}:=\{G \times V \mid V \in \mathcal{V}\}$ then $\mathcal{U}$ is an $\mathcal{F}$-cover for $G \times X$ of dimension $N$ and $G \times\{x\} \subseteq U=G \times V \in \mathcal{V}$ whenever $x \in V \in \mathcal{V}$. Such a cover $\mathcal{V}$ exists for example for any cellular action on an $N$-dimensional $C W$-complex whenever all isotropy groups of the action belong to $\mathcal{F}$

Remark 0.4. Suppose that $X$ is compact and metrizable. Then given an $N$ - $\mathcal{F}$ amenable action of a countable group $G$ on $X$ there is a sequence of $G$-equivariant 
maps $f_{n}: G \times X \rightarrow K_{n}$ with the following properties. The space $K_{n}$ is an $N$ dimensional simplicial complex with a simplicial $G$-action. The isotropy groups for this action belong to $\mathcal{F}$. The maps $f_{n}$ are contracting in the $G$-direction: for any $g \in G$ we have $\sup _{x \in X}\left\|f_{n}(e, x)-f_{n}(g, x)\right\|_{1} \rightarrow 0$ as $n \rightarrow \infty$. The maps $f_{n}$ can be contructed using a partition of unity subordinated to the covers $\mathcal{U}$ appearing in the definition of $N-\mathcal{F}$-amenability, compare 6. Sec. 4 and 5]. The maps $f_{n}(e,-): X \rightarrow K_{n}$ are then almost $G$-equivariant in the following sense: for any $g \in G$ we have $\sup _{x \in X}\left\|g f_{n}(e, x)-f_{n}(e, g x)\right\|_{1} \rightarrow 0$ as $n \rightarrow \infty$. The action of $G$ on $X$ is amenable 34 if there is such an almost equivariant sequence of maps to the space of probability measures on $G$. The isotropy groups for the action on the space of probability measures are the finite subgroups of $G$. Thus $N$ - $\mathcal{F}$-amenability can be thought of as both stronger and weaker than amenabilty: stronger since a finite dimensional target is required; weaker since the target may have larger isotropy groups.

Ozawa 35 investigated amenable actions for relatively hyperbolic groups. In particular, his results imply that if $G$ is relatively hyperbolic to amenable groups, then the action of $G$ on the boundary is amenable.

Flow spaces. If $G$ is the fundamental group of a negatively curved manifold $M$, then the geodesic flow is a flow on the unit sphere bundle $S M$ of the tangent bundle of $M$. We will say that $S M$ together with the geodesic flow is the geodesic flow space for $G$. Let $G$ now be a hyperbolic group. Mineyev [29] constructed an analog of the geodesic flow space and this flow space $F S$ and its dynamic properties are key ingredients to the proof of finite VCyc-amenability for the action of $G$ on its boundary in [5]. The proof has naturally two parts. In the first part the so called long and thin covers of $F S$ are constructed. In the second part the dynamic of the flow is used to construct maps $G \times \partial G \rightarrow F S$ under which the long and thin covers of $F S$ pull back to the necessary covers of $G \times \partial G$.

The key property of the long and thin covers $\mathcal{U}_{\alpha}$ is that they are long in the direction of the flow: for each $x \in F S$ there is $U \in \mathcal{U}_{\alpha}$ such that $x$ stays in $U$ for time $t \in[-\alpha, \alpha]$. Typically the members of $\mathcal{U}$ are very thin transverse to the flow - thus the name long and thin covers. These covers are a variation of the long and thin cell structures appearing in [19. The construction of these long and thin covers in 5] is quite involved but works for very general flow spaces. Moreover, assumptions on the order of finite subgroups of $G$ and the structure of periodic orbits were later shown to be not necessary by Mole-Rüping 32 and by Kasprowski-Rüping [26. Sauer 38] used packing methods to prove in a measure theoretic context results that are similar to long and thin covers. Later he pointed out that such packing methods should also be applicable to the construction of long and thin covers of flow spaces. This led to a much simpler construction for the long and thin covers from [5] by Kasprowski-Rüping [26].

Coarse flow spaces. An observation of the present paper is that the construction of long and thin covers using the packing method works in a more general context than flow spaces. This can be used to give an alternative argument for the finitely VCyc-amenability of the actions of hyperbolic groups on their boundaries that avoids Mineyev's flow space. Moreover, this alternative argument generalizes to relatively hyperbolic group 1 . In this and the next subsection we outline this argument for hyperbolic groups. The case of relatively hyperbolic groups is treated in detail in the main text of this paper.

\footnotetext{
${ }^{1}$ It is plausible that the argument from $[5$ can also be extended to relatively hyperbolic groups. A step in this direction is 31 .
} 
Let $G$ be a hyperbolic group. We will replace Mineyev's flow space with a more easily defined coarse flow space. Let $\Gamma$ be a Cayley graph for $G$. Assume that $\Gamma$ is $\delta$-hyperbolic. Let $\bar{G}:=G \cup \partial G$ and $Z:=\bar{G}^{2}$.

Definition 0.5. The coarse flow space $C F$ for $G$ is the subspace of $G \times Z$ consisting of triples $\left(g, \xi_{-}, \xi_{+}\right)$such that there exists a geodesic in $\Gamma$ from $\xi_{-}$to $\xi_{+}$that passes $g$ within distance $\delta$.

There is no actual flow on this coarse flow space, but there are natural analoga of the orbits of the flow on $F S$. These analoga are the subsets $G_{\xi_{-}, \xi_{+}}:=\{g \in$ $\left.G \mid\left(g, \xi_{-}, \xi_{+}\right) \in C F\right\} \subseteq G$. Since $\Gamma$ is hyperbolic each $G_{\xi_{-}, \xi_{+}}$is contained in a uniformly bounded neighborhood of a geodesic. Consequently, the $G_{\xi_{-}, \xi_{+}}$satisfy a uniform doubling property: there is $D$ such that for any $R$ and any subset $S$ of a $2 R$-ball in $G_{\xi_{-}, \xi_{+}}$the following holds: if $S$ is $R$-separated, i.e., $d_{G}\left(s, s^{\prime}\right) \geq R$ for all $s \neq s^{\prime} \in S$, then the cardinality of $S$ is at most $D$. This observation is the main ingredient for the following version of long and thin covers for $C F$. Let $d_{G}$ be a left-invariant word metric on $G$.

Proposition 0.6. There is $N$ such that for any $\alpha>0$ there exists an VCyc-cover $\mathcal{W}$ of $C F$ such that the following holds:

a) for any $\left(g, \xi_{-}, \xi_{+}\right) \in C F$ there is $W \in \mathcal{W}$ such that $B_{\alpha}(g) \times\left\{\left(\xi_{-}, \xi_{+}\right)\right\} \cap C F \subseteq$ $W$;

b) the dimension of $\mathcal{W}$ is at most $N$.

In Theorem 1.1 we prove a version of this result in a more general situation that will also be applicable to the coarse flow spaces for relatively hyperbolic groups introduced in Definition 3.4. An important assumption is again a uniform doubling property. As an application of Theorem 1.1 we obtain a version of Proposition 0.6 for relatively hyperbolic groups in Proposition 3.7. In a different direction a corollary to Theorem 1.1 is that all actions of finitely generated virtually nilpotent groups on finite dimensional normal separable spaces with isotropy in $\mathcal{F}$ are finitely $\mathcal{F}$-amenable, see Corollary 1.10. This generalizes a result for free actions of nilpotent groups by Szabó-Wu-Zacharis 39 .

Sketch of proof of Proposition [0.6] if $\operatorname{dim} \partial G=0$. Since $\operatorname{dim} \bar{G}^{2}=0$ there is a basis of the topology of $\bar{G}^{2}$ consisting of sets that are open and closed. Choose a covering of $C F$ by sets of the form $\left\{g_{i}\right\} \times V_{i} \cap C F, i \in \mathbb{N}$ where $V_{i}$ is open and closed in $\bar{G}^{2}$. Then define inductively $U_{i}$ by $U_{0}:=V_{0}$ and

$$
U_{i}:=V_{i} \backslash \bigcup_{j} U_{j}
$$

where the union is over all $j$ with $j<i$ and $d_{G}\left(g_{i}, g_{j}\right) \leq \alpha$. Since the $V_{i}$ are closed and open, the $U_{i}$ are still open. The open sets $W_{i}:=B_{2 \alpha}\left(g_{i}\right) \times U_{i} \cap C F$ form then the desired cover $\mathcal{W}$ of $C F$. This cover is $\alpha$-long in the direction of $G$, more or less by construction. To compute the dimension of $\mathcal{W}$ one checks that if $W_{i_{1}} \cap \cdots \cap W_{i_{N}} \neq \emptyset$, then the $g_{i}$ form an $\alpha$-separated set in a ball of radius $2 \alpha$ in one of the $G_{\xi_{-}, \xi_{+}}$and therefore $\operatorname{dim} \mathcal{W} \leq D-1$.

In this sketch we ignored the action of $G$ on $C F$. To amend this one has to choose the $V_{i}$ sufficiently small in order to avoid intersections $g W_{i} \cap W_{i}$ for to many $g$. Moreover, in the definition of $U_{i}$ and $W_{i}$ the group action has to be taken into account. In order to extend the argument to the case $\operatorname{dim} \partial G>0$ an induction over subspaces of $C F$ of lower dimension can be used. 
Pulling back long and thin covers from $C F$ to $G \times \partial G$. Let $C F$ be the coarse flow space for the hyperbolic group $G$. For $W \subseteq C F$ and $\tau>0$ we define $\iota^{-\tau} W \subseteq$ $G \times \partial G$ to consist of all pairs $(g, \xi)$ with the following property. If $v \in G$ belongs to a geodesic from $g$ to $\xi$ and satisfies $d_{G}(g, v)=\tau$, then $(v, g, \xi) \in W$.

One way to think about $\iota^{-\tau} W$ is as follows: First define $\iota: G \times \partial G \rightarrow C F$ by $\iota(g, \xi)=(g, g, \xi)$. Next apply a partially defined multi-valued geodesic flow $\phi_{\tau}$ on $C F$ : this flow takes $(g, g, \xi)$ to the set of all $(g, v, \xi)$ where $v \in G$ belongs to a geodesic between $g$ and $\xi$ and is of distance $\tau$ from $g$. Then $\iota^{-\tau} W$ is the pull-back of $W$ under the composition $\phi_{\tau} \circ \iota$.

This construction allows us to use the long thin covers of $C F$ from Proposition 0.6 to prove that the action of $G$ on $\partial G$ is finitely VCyc-amenable: If $\mathcal{W}$ is a VCyccover of $C F$ then the same holds for $\iota^{-\tau} \mathcal{W}:=\left\{\iota^{-\tau} W \mid W \in \mathcal{W}\right\}$. By construction $\operatorname{dim} \iota^{-\tau} \mathcal{W} \leq \operatorname{dim} \mathcal{W}$. Finally, if $\mathcal{W}$ is a long cover of $C F$ (as in Proposition 0.6 a) then for sufficiently large $\tau$ the cover $\iota^{-\tau} \mathcal{W}$ of $G \times \partial G$ is wide in the $G$-direction (as in Definition 0.1 b) . This last fact can be thought of as a consequence of dynamic properties of $\phi_{\tau}$ and uses the hyperbolicity of $\Gamma$.

For relatively hyperbolic groups this step is carried out in detail in Section 3 . The main additional difficulty appearing is discussed in the next subsection.

Relatively hyperbolic groups. The precise definitions for relatively hyperbolic groups that we use will be given in Section 2 and mostly follows Bowditch 13 . Let $G$ be relatively hyperbolic to the peripheral subgroups $P_{1}, \ldots, P_{n}$. By definition $G$ acts on a hyperbolic graph $\Gamma$. Unlike the Cayley graph for hyperbolic groups $\Gamma$ will contain vertices of infinite valency and the isotropy groups of these vertices will be conjugated to the $P_{i}$. We write $V$ for the set of vertices of $\Gamma$ and $V_{\infty}$ for the set of vertices of $\Gamma$ of infinite valency. The boundary of $G$ is defined by Bowditch as the union of $\Delta:=\partial \Gamma \cup V_{\infty}$; this is a compact space.

The key additional property of the graph $\Gamma$ used here is fineness, as introduced by Bowditch [13. Under a mild additional assumption this property can be used to define a proper metric on the set of edges of $\Gamma$, see 30. In particular, it is possible to measure angles in $\Gamma$. Here an angle is a pair of edges that share a vertex. In order to allow for peripheral subgroups that are not necessarily finitely generated it is better to avoid the additional assumption. To this end we take a slightly different point of view and consider $G$-invariant $G$-cofinite subsets $\Theta$ of the set of all angles. Such a subset will be called a size for angles. Each size for angles $\Theta$ is then locally finite in the following sense: for each edge $e$ there are only finitely many edges $e^{\prime}$ such that $\left(e, e^{\prime}\right) \in \Theta$. Fineness of $\Gamma$ implies that the set of all angles appearing in any non-degenerate geodesic triangle is such a size for angles.

For any size for angles $\Theta$ we define a coarse flow space $C F(\Theta)$. Its definition is similar to the hyperbolic case in Definition 0.5 . where we replace $G$ with vertices of finite valency and only use geodesics along which all angles are $\Theta$-small. The argument outlined in the hyperbolic case above can then be used to produce wide covers of a certain subspace of $G \times \Delta$, see Proposition 3.2. In order to prove that the action of $G$ on $\Delta$ is finitely $\mathcal{P}$-amenable, we need to extend these wide covers to all of $G \times \Delta$. This is done by an explicit construction in Proposition 3.17 .

Acknowledgements. I thank Adam Mole for many discussions about relatively hyperbolic groups, Roman Sauer and Daniel Kasprowski for discussions about long and thin covers and packing methods, Brian Bowditch and François Dahmani for helpful emails about relatively hyperbolic groups and their boundaries. Comments by Svenja Knopf, Timm von Puttkamer and a referee greatly improved this paper. This work has been supported by the SFB 878 in Münster. 


\section{LONG THIN COVERS OF SUBSPACES OF $V \times Z$}

Throughout this section we fix

- a group $G$;

- a family $\mathcal{F}$ of subgroups of $G$;

- a discrete countable proper metric space $V$ with a proper isometric $G$-action, the metric of $V$ will be denoted by $d_{V}$; we allow distances for $d_{V}$ to be $\infty$;

- a separable metrizable space $Z$ with an action of $G$ by homeomorphisms;

- a closed $G$-invariant subspace $X$ of $V \times Z$; we will always use the diagonal action of $G$ on $V \times Z$.

An example for $X$ is the coarse flow space for hyperbolic groups from Definition 0.5. Let $\alpha>0$. We write $B_{\alpha}(v):=\left\{w \in V \mid d_{V}(v, w) \leq \alpha\right\}$ for the closed $\alpha$-ball around $v$. A subset $S \subseteq V$ is said to be $\alpha$-separated if $d_{V}\left(s, s^{\prime}\right)>\alpha$ for any two distinct elements of $S$. We will say that a subset $V_{0}$ of $V$ has the $(D, R)$ doubling property if for any $\alpha \geq R$ the following holds: if $S \subseteq V_{0}$ is $\alpha$-separated and contained in a ball of radius $2 \alpha$, then the cardinality of $S$ is at most $D$.

For $v \in V$ we set $Z_{v}:=\{z \in Z \mid(v, z) \in X\}$. This is a closed subset of $Z$. For $z \in Z$ we set $V_{z}:=\{v \in V \mid(v, z) \in X\} \subseteq V$. Then

$$
X=\bigcup_{v \in V}\{v\} \times Z_{v}=\bigcup_{z \in Z} V_{z} \times\{z\} .
$$

The following is the most abstract result about long thin covers in this paper.

Theorem 1.1. Assume that $X$ satisfies the following assumptions.

A) $X$ is finite dimensional;

$B)$ there are $D>0, R \geq 0$ such that for all $z \in Z$ the subspace $V_{z}$ of $V$ has the $(D, R)$-doubling property;

$C)$ for each $(v, z) \in X$ the isotropy group $G_{z}:=\{g \in G \mid g z=z\}$ belongs to $\mathcal{F}$. Then $X$ admits long thin covers as follows: there is a number $N$ depending only on the dimension of $X$ and the doubling constant $D$, such that for any $\alpha>0$ there is an $\mathcal{F}$-cover $\mathcal{W}$ of $X$ such that

a) $\operatorname{dim} \mathcal{W} \leq N$

b) for any $(v, z) \in X$ there is $W \in \mathcal{W}$ such that $B_{\alpha}(v) \times\{z\} \cap X \subseteq W$.

The proof of Theorem 1.1 will proceed by induction on the dimension of subspaces of $X$. The following proposition is the induction step. Since we can take $Y=X$ to start the induction it implies Theorem 1.1

Proposition 1.2. Retain the assumptions of Theorem [1.1. Let $Y \subseteq X$ be a nonempty $G$-invariant closed subspace. Assume that $\operatorname{dim} Y=n$. For any $\alpha>0$ there is a $G$-invariant collection $\mathcal{W}$ of $\mathcal{F}$-subsets of $X$ and a $G$-invariant closed subspace $Y^{\prime} \subseteq Y$ such that

a) $\operatorname{dim} Y^{\prime}<\operatorname{dim} Y$;

b) the order of $\mathcal{W}$ is at most $D-1$;

c) for any $(v, z) \in Y \backslash Y^{\prime}$ there is $W \in \mathcal{W}$ such that $B_{\alpha}(v) \times\{z\} \cap X \subseteq W$.

Lemma 1.3. Retain the assumptions of Proposition 1.2. Let $(v, z) \in Y, \alpha>0$ and $U_{0}$ be a neighborhood of $z$ in $Z$. Then $U_{0}$ contains a smaller open neighborhood $U$ of $z$ in $Z$ such that

a) $\left\{g \in G \mid d_{V}(g v, v) \leq \alpha, U \cap g U \neq \emptyset\right\} \subseteq G_{z}=\{g \in G \mid g z=z\}$;

b) for all $w \in V$ we have $\operatorname{dim} Y_{w} \cap \partial U<\operatorname{dim} Y$, where $Y_{w}:=\left\{z \in Z_{w} \mid(w, z) \in\right.$ $Y\}$ and $\partial U$ denotes the boundary of $U$ in $Z$.

Proof. We have $Y=\bigcup_{w \in V}\{w\} \times Y_{w}$. Since $\operatorname{dim} Y=n$ and $V$ is discrete each $Y_{w}$ satisfies $\operatorname{dim} Y_{w} \leq n$. Since $Y$ is closed, $Y_{w} \subseteq Z$ is closed for each $w \in V$. The 
countable sum theorem in dimension theory [36, Thm. 2.5, p.125] asserts that in a normal space the dimension of the countable union of closed subspaces is the supremum of the dimension of the subspace. Since $V$ is countable this implies that the dimension of $Y_{V}:=\bigcup_{w \in V} Y_{w}$ is at most $n$.

Since every subspace $Z^{\prime}$ of $Z$ is separable and metrizable, its (covering) dimension equals its (small) inductive dimension: $\operatorname{dim} Z^{\prime}=\operatorname{ind} Z^{\prime}$ [36, Cor. 5.10, p. 184]. This means that for any $z \in Z^{\prime}$ there are arbitrary small neighborhoods whose boundary in $Z^{\prime}$ has dimension less than $\operatorname{dim} Z^{\prime}$.

Since $d_{V}$ is proper and the action of $G$ on $V$ is proper, $\left\{g \mid d_{V}(g v, v) \leq \alpha\right\}$ is finite. Since $Z$ is Hausdorff there is an open neighborhood $U^{\prime}$ of $z$ in $Z$ that is contained in $U_{0}$ and satisfies $g U^{\prime} \cap U^{\prime}=\emptyset$ for all $g \in G \backslash G_{z}$ with $d_{V}(g v, v) \leq$ $\alpha$. Applying the facts from the preceding paragraph to $Y_{V}$, we obtain an open neighborhood $U^{\prime \prime}$ of $z$ in $Y_{V}$ that is contained in $U^{\prime}$ and satisfies $\operatorname{dim} \partial^{Y_{V}} U^{\prime \prime}<$ $\operatorname{dim} Y$, where $\partial^{Y_{V}}$ denotes the boundary in $Y_{V}$. Using Lemma B.1 we can extend $U^{\prime \prime}$ to an open subset $U$ of $Z$ that is contained in $U^{\prime}$ and satisfies $U \cap Y_{V}=U^{\prime \prime}$, and $\partial U \cap Y_{V}=\partial^{Y_{V}} U^{\prime \prime}$, where $\partial$ denotes the boundary in $Z$.

Let $w \in V$. Since $Y_{w}$ is closed in $Y_{V}$ we have $\operatorname{dim} Y_{w} \cap \partial U \leq \operatorname{dim} Y_{V} \cap \partial U=$ $\operatorname{dim} \partial^{Y_{V}} U^{\prime \prime}<\operatorname{dim} Y$. Thus $U$ satisfies b) Since $U \subseteq U^{\prime}, U$ also satisfies a).

Lemma 1.4. Retain the assumptions of Theorem 1.1. Let $\alpha \geq R$ be given. Then there exists an open $G$-invariant neighborhood $X^{\text {th }}$ of $X$ in $V \times Z$ such that for all $z \in Z$, the subspace $V_{z}^{\text {th }}:=\left\{v \in V \mid(v, z) \in X^{\text {th }}\right\}$ of $V$ has the following property: if $S \subseteq V_{z}^{\text {th }}$ is $\alpha$-separated and contained in a ball of radius $2 \alpha$, then the cardinality of $S$ is at most $D$.

Proof. Since the action of $G$ on $V$ is proper, we can pick metrics $d_{v}, v \in V$ on $Z$ that are compatible with the $G$-action, i.e., such that we have $d_{v}\left(z, z^{\prime}\right)=d_{g v}\left(g z, g z^{\prime}\right)$ for all $v \in V, g \in G, z, z^{\prime} \in Z$.

Let $(v, z) \in X$ (i.e., $z \in Z_{v}$ ). For $w \in V$ with $z \notin Z_{w}$, let $\varepsilon(v, w, z):=$ $d_{v}\left(z, Z_{w}\right) / 3$. The $Z_{w}$ are closed since $X$ is closed. Thus $\varepsilon(v, w, z)>0$. Let $\varepsilon(v, z):=$ $\min \varepsilon(v, w, z)$, where the minimum is taken over all $w \in V$ with $d_{V}(v, w) \leq 4 \alpha$ and $z \notin Z_{w}$. Since $d_{V}$ is proper the minimum exists and is positive. Let $B(v, w, z)$ be the open ball of radius $\varepsilon(v, z)$ around $z$ with respect to the metric $d_{w}$. Set $B(v, z):=\cap_{d_{V}(v, w) \leq 4 \alpha} B(v, w, z)$. Since $d_{V}$ is proper $B(v, z)$ is open. Moreover, by construction,

(1.5) $d_{v}\left(z, Z_{w}\right)>2 \varepsilon(v, z)$ whenever $z \in Z_{v} \backslash Z_{w}$ and $d_{V}(v, w) \leq 4 \alpha$;

(1.6) $d_{v}\left(z^{\prime}, z\right)<\varepsilon(w, z)$ whenever $z \in Z_{w}, z^{\prime} \in B(w, z)$ and $d_{V}(v, w) \leq 4 \alpha$.

Define $Z_{v}^{t h}:=\bigcup_{z \in Z_{v}} B(v, z)$ and $X^{t h}:=\bigcup_{v \in V}\{v\} \times Z_{v}^{t h}$. By construction $X^{t h}$ is an open neighborhood of $X$ in $V \times Z$. The compatibility of the metrics $d_{v}$ with the $G$-action guarantees that $X^{\text {th }}$ is $G$-invariant.

Let $S \subseteq V$ be an $\alpha$-separated subset of some $2 \alpha$-ball in $V$. Assume that the cardinality of $S$ exceeds $D$. Since all $V_{z}$ have the $D$-doubling property we have $\bigcap_{w \in S} Z_{w}=\emptyset$. We need to show that $\bigcap_{w \in S} Z_{w}^{t h}=\emptyset$ as well. Assume by contradiction that $z^{\prime} \in \bigcap_{w \in S} Z_{w}^{t h}$. Then we find for each $w \in S$, a point $z_{w} \in Z_{w}$ with $z^{\prime} \in B\left(w, z_{w}\right)$. Choose $v \in S$ such that $\varepsilon\left(w, z_{w}\right) \leq \varepsilon\left(v, z_{v}\right)$ for all $w \in S$. Note that $d_{V}(w, v) \leq 4 \alpha$ for all $w \in S$. Now (1.6) implies that for all $w \in S$

$$
\begin{aligned}
d_{v}\left(z_{v}, Z_{w}\right) \leq d_{v}\left(z_{v}, z_{w}\right) \leq d_{v}\left(z_{v}, z^{\prime}\right) & +d_{v}\left(z^{\prime}, z_{w}\right) \\
& \leq \varepsilon\left(v, z_{v}\right)+\varepsilon\left(w, z_{w}\right) \leq 2 \varepsilon\left(v, z_{v}\right) .
\end{aligned}
$$

On the other hand (1.5) implies $d_{v}\left(z_{v}, Z_{w}\right)>2 \varepsilon\left(v, z_{v}\right)$ if $z_{v} \notin Z_{w}$. Therefore $z_{v} \in Z_{w}$ for all $w \in S$. This contradicts $\bigcap_{w \in S} Z_{w}=\emptyset$.

Proof of Proposition 1.2. Throughout this proof closure and boundary will always be taken with respect to $Z$. 
Let $\alpha \geq R$. Let $X^{\text {th }}$ be as in Lemma 1.4. Since $Y$ is separable we can use Lemma 1.3 and find sequences $v_{i} \in V, F_{i} \in \mathcal{F}, U_{i} \subseteq Z$ open, such that

(1.7) $\left\{g \in G \mid d_{V}\left(g v_{i}, v_{i}\right) \leq 4 \alpha, U_{i} \cap g U_{i} \neq \emptyset\right\} \subseteq F_{i}$;

(1.8) for all $w \in V$ we have $\operatorname{dim} Y_{w} \cap \partial U_{i}<\operatorname{dim} Y$;

(1.9) $Y \subseteq \bigcup_{i}\left\{v_{i}\right\} \times U_{i} \subseteq X^{t h}$

Now we define inductively $U_{i}^{\prime}$ by $U_{0}^{\prime}:=U_{0}$ and

$$
U_{i}^{\prime}:=U_{i} \backslash \bigcup_{h, j} h \overline{U_{j}^{\prime}}
$$

where the union is over all pairs $(h, j)$ with $h \in G, j<i$, and $d_{V}\left(v_{i}, h v_{j}\right) \leq \alpha$. Since $d_{V}$ is proper and the action of $G$ on $V$ is proper, this is a finite set of pairs and $U_{i}^{\prime}$ is open. An easy induction shows that $\partial U_{i}^{\prime} \subseteq \bigcup_{j<i, g \in G} g \partial U_{j}$. Thus with $Y^{\prime \prime}:=Y \cap V \times \bigcup_{i, g} g \partial U_{i}$ we have

$$
Y \cap\{v\} \times g \partial U_{i}^{\prime} \subseteq Y^{\prime \prime}
$$

for all $v \in V, i \in \mathbb{N}, g \in G$. For $i \in \mathbb{N}$ let

$$
W_{i}:=B_{2 \alpha}\left(v_{i}\right) \times U_{i}^{\prime} \cap X
$$

and set $\mathcal{W}:=\left\{g F_{i} W_{i} \mid g \in G, i \in \mathbb{N}\right\}$.

Clearly, $\mathcal{W}$ consists of open subsets of $X$ and is $G$-invariant. Consider $g F_{i} W_{i} \in$ $\mathcal{W}$. If $\gamma \in g F_{i} g^{-1}$, then $\gamma g F_{i} W_{i}=g F_{i} W_{i}$. If $\gamma \notin g F_{i} g^{-1}$, then $\gamma g F_{i} W_{i} \cap$ $g F_{i} W_{i}=\emptyset$. Indeed, if $(v, z) \in \gamma g F_{i} W_{i} \cap g F_{i} W_{i}$, then there are $a, b \in F_{i}$ with $d_{V}\left(v, \gamma g a v_{i}\right), d_{V}\left(v, g b v_{i}\right) \leq 2 \alpha$ and $z \in \gamma g a U_{i} \cap g b U_{i}$. Then (1.7) implies $b^{-1} g^{-1} \gamma g a \in$ $F_{i}$ and therefore $\gamma \in g F_{i} g^{-1}$. Thus each $g F_{i} W_{i}$ is an $\mathcal{F}$-subset.

We now prove $\operatorname{dim} Y^{\prime \prime}<\operatorname{dim} Y$. For $v \in V, i \in \mathbb{N}$ and $g \in G$ we have $Y \cap$ $\{v\} \times g \partial U_{i}=g\left(Y \cap\left\{g^{-1} v\right\} \times \partial U_{i}\right) \cong Y_{g^{-1} v} \cap \partial U_{i}$. By (1.8) all these spaces are of dimension $<\operatorname{dim} Y$. Since they are all closed in $Y$ the countable sum theorem 36 , Thm. 2.5, p.125] implies that their union, $Y^{\prime \prime}$, is also of dimension $<\operatorname{dim} Y$.

Next we prove that $\mathcal{W}$ is of order $\leq D-1$, i.e., that it satisfies $\mathrm{b})$, Let $(v, z) \in X$. Suppose that $(v, z) \in g F_{i} W_{i}$. Then there is $a \in F_{i}$ such that $d_{V}\left(v, g a v_{i}\right) \leq 2 \alpha$ and $z \in g a U_{i}^{\prime}$. As $X^{\text {th }}$ is $G$-invariant, (1.9) implies $\left(\right.$ gav $\left._{i}, z\right) \in\left\{g a v_{i}\right\} \times\left\{g a U_{i}^{\prime}\right\} \subseteq X^{t h}$. Thus $\operatorname{gav}_{i} \in V_{z}^{t h}$. If also $(v, z) \in h F_{j} W_{j}$ then there is $b \in F_{j}$ with $d_{V}\left(v, h b v_{j}\right) \leq 2 \alpha$, $z \in h b U_{j}^{\prime}$ and $h b v_{j} \in V_{z}^{\text {th }}$.

If $i=j$ then $g F_{i} W_{i} \cap h F_{i} W_{i} \neq \emptyset$ and since $g F_{i} W_{i}$ is a $\mathcal{F}$-subset we then have $g F_{i} W_{i}=h F_{j} W_{j}$. If $i \neq j$, then $\gamma U_{i}^{\prime} \cap U_{j}^{\prime}=\emptyset$ for all $\gamma \in G$ with $d_{V}\left(\gamma v_{i}, v_{j}\right) \leq \alpha$. Since $b^{-1} h^{-1} g a U_{i}^{\prime} \cap U_{j}^{\prime} \neq \emptyset$, this implies $d_{V}\left(b^{-1} h^{-1} \operatorname{gav}_{i}, v_{j}\right)=d_{V}\left(\right.$ gav $\left._{i}, h b v_{j}\right)>\alpha$.

All together, we have shown that if $(v, z)$ is contained in $N$ distinct members in $\mathcal{W}$, then there is an $\alpha$-separated set in $B_{2 \alpha}(v) \cap V_{z}^{t h}$ of cardinality $N$. Lemma 1.4 implies now that $N$ is bounded by the doubling constant $D$ appearing in the assumptions of Theorem 1.1. This implies that the order of $\mathcal{W}$ is at most $D-1$.

Next we claim that for any $(v, z) \in Y \backslash Y^{\prime \prime}$ there is $W \in \mathcal{W}$ with $B_{\alpha}(v) \times\{z\} \cap X \subseteq$ $W$. Let $(v, z) \in Y \backslash Y^{\prime \prime}$. By (1.9) there is $i \in \mathbb{N}$ with $(v, z) \in\left\{v_{i}\right\} \times U_{i}$. So $v=v_{i}$. If $z \in U_{i}^{\prime}$, then

$$
B_{\alpha}(v) \times\{z\} \cap X \subseteq B_{2 \alpha}\left(v_{i}\right) \times U_{i}^{\prime} \cap X \subseteq F_{i} W_{i} .
$$

If $z \notin U_{i}^{\prime}$, then $z \in h \bar{U}_{j}^{\prime}$ for some $j<i$ and $h \in G$ with $d_{V}\left(v_{i}, h v_{j}\right) \leq \alpha$. We observed earlier $Y \cap\{v\} \times h \partial U_{j}^{\prime} \subseteq Y^{\prime \prime}$. Thus we have $z \in h U_{j}^{\prime}$. Since $d_{V}\left(v, h v_{j}\right)=$ $d_{V}\left(v_{i}, h v_{j}\right) \leq \alpha$ we have then

$$
B_{\alpha}(v) \times\{z\} \cap X \subseteq B_{2 \alpha}\left(h v_{j}\right) \times h U_{j}^{\prime} \cap X \subseteq h F_{j} W_{j}
$$

This proves our claim. 
Now observe that if $B_{\alpha}(v) \times\{z\} \cap X \subseteq W$, then, since $W$ is open, $B_{\alpha}(v) \times\left\{z^{\prime}\right\} \cap$ $X \subseteq W$ for all $z^{\prime}$ in a neighborhood of $z$. Since $\mathcal{W}$ is $G$-invariant, this implies that there is an open $G$-invariant neighborhood $N$ of $Y \backslash Y^{\prime \prime}$ such that all $(v, z) \in N$ satisfy c)

Now $Y^{\prime}:=Y \backslash N$ is $G$-invariant and closed. Moreover, since $Y^{\prime} \subseteq Y^{\prime \prime}$, we have $\operatorname{dim} Y^{\prime} \leq \operatorname{dim} Y^{\prime \prime}<\operatorname{dim} Y$ and a) holds.

Corollary 1.10. Let $G$ be a finitely generated virtually nilpotent group. Then any action of $G$ on a finite dimensional separable metrizable space $Z$ is finitely $\mathcal{F}$-amenable where $\mathcal{F}$ consists of all subgroups of $G$ that fix a point in $Z$.

Proof. This follows from Theorem 1.1 where we take $V=G$ with a word metric and $X=G \times Z$ : Assumption A) is satisfied since $Z$ is finitely dimensional. Finitely generated virtually nilpotent groups are of polynomial growth [10. This implies that $G$ has a doubling property. Thus assumption B) is satisfied. Assumption C) holds by choice of $\mathcal{F}$.

\section{Relative hyperbolic Groups}

Throughout this section $\Gamma$ will be a fine and hyperbolic graph in the sense of Bowditch [13] and $G$ will be a countable group equipped with a cocompact simplicial action on $\Gamma$. In particular, $\Gamma$ is uniformly fine: for any $\alpha$ there is $N_{\alpha}$ such that for any edge $e$ there are at most $N_{\alpha}$ circuits of length $\leq \alpha$ containing $e$. (A circuit is an embedded loop in $\Gamma$.) The isotropy groups of edges for the action of $G$ on $\Gamma$ are assumed to be finite. Let $P_{1}, \ldots, P_{n}$ be representatives of the conjugacy classes of the isotropy groups of the vertices of $\Gamma$ of infinite valency. We will then say that $G$ is relatively hyperbolic to $P_{1}, \ldots, P_{n} 2$.

We denote by $\Delta$ the union of the Gromov boundary $\partial \Gamma$ of $\Gamma$ with the set $V_{\infty}$ of vertices of $\Gamma$ with infinite valency. By $V$ we denote the set of all vertices of $\Gamma$ and by $E$ the set of all edges of $\Gamma$. We write $\Delta_{+}:=\partial \Gamma \cup V$. In other words, $\Delta_{+}$is the union of $\Delta$ with the set of all vertices of $\Gamma$ of finite valency. We think of edges as subsets of $V$ with two elements; in particular, edges are not oriented.

We will use the term geodesic for finite geodesics, geodesic rays and bi-infinite geodesics. If $c$ is a geodesic and $\xi, \xi^{\prime} \in \Delta_{+}$are both contained in $c$ or endpoints of $c$ then we write $\left.c\right|_{\left[\xi, \xi^{\prime}\right]}$ for the restriction of $c$ to a geodesic between $\xi$ and $\xi^{\prime}$.

Hyperbolicity of a graph is usually formulated in terms of geodesic triangles whose sides are finite geodesics. But it then follows that all geodesic triangles, including geodesic triangles with one or more corners at the boundary are uniformly slim [24, Lem. 2.113. We can therefore fix a constant $\delta>0$ such that in all geodesic triangles each side is contained in the union of the $\delta$-neighborhood of the other two. It will be convenient to assume that $\delta$ is an integer. We will refer to $\delta$ as a hyperbolicity constant for $\Gamma$.

Angles. An unordered pair $\left(e, e^{\prime}\right)$ of edges in $\Gamma$ that have a vertex $v$ in common is called an angle at $v$. If $e=e^{\prime}$, then we say that the angle $\left(e, e^{\prime}\right)$ is trivial. The group $G$ acts on the set of angles; this action will usually be not cofinite. A $G$-invariant, $G$-cofinite subset $\Theta$ of the set of all angles, that contains all trivial angles will be called a size for angles. Members of $\Theta$ will be called $\Theta$-small. Angles that are not contained in $\Theta$ will be called $\Theta$-large. If $c$ is a geodesic in $\Gamma$ then $c$ determines a non-trivial angle at every internal vertex $v$ of $c$; this angle will be called the angle

\footnotetext{
${ }^{2}$ Bowditch [13, Def. 2] assumes in addition that $P_{1}, \ldots, P_{n}$ are finitely generated, but this restriction will not be necessary here.

${ }^{3}$ In this reference the proof is left as an exercise; for completeness a solution to this exercise is included in Appendix $\mathrm{C}$
} 
of $c$ at $v$ and sometimes be denoted by $\Varangle_{v} c$. If all these angles are $\Theta$-small, then we will say that $c$ is $\Theta$-small. In particular, geodesics of length 1 are always $\Theta$-small, as they contain no internal vertices. If $\Theta$ and $\Theta^{\prime}$ are two sizes for angles then we define $\Theta+\Theta^{\prime}$ to consist of all angles $\left(e, e^{\prime \prime}\right)$ for which there is an edge $e^{\prime}$ with $\left(e, e^{\prime}\right) \in \Theta$ and $\left(e^{\prime}, e^{\prime \prime}\right) \in \Theta^{\prime}$. As a consequence of the following Lemma 2.1. $\Theta+\Theta^{\prime}$ is again a size for angles.

Lemma 2.1. If $\Theta$ is a size for angles then each edge $e$ is contained in only finitely many angles of $\Theta$, i.e., $\Theta_{e}:=\left\{e^{\prime} \in E \mid\left(e, e^{\prime}\right) \in \Theta\right\}$ is finite. Conversely, $a G$ invariant set of angles $\Theta$ with the property that $\Theta_{e}$ is finite for any edge e is a size for angles.

Proof. If the action of $G$ on $\Theta$ is cofinite, the same holds for the action of the isotropy group $G_{e}$ of $e$ on $\Theta_{e}$. Since $G_{e}$ is finite, so is then $\Theta_{e}$.

Conversely, since the action of $G$ on $E$ is cofinite, there are finitely many edges $e_{1}, \ldots, e_{n}$ such that each orbit for the action of $G$ on the set of all angles contains an angle of the form $\left(e, e_{i}\right)$. Thus, if $\Theta_{e_{i}}$ is finite for $i=1, \ldots, n$, then the action of $G$ on $\Theta$ is cofinite.

Corollary 2.2. Let $\Theta$ be a size for angles and $\alpha>0$. Let $v$ be a vertex and $e$ be an edge incident to $v$. Consider the set $V^{\prime}$ of all vertices $v^{\prime}$ for which there exists $a \Theta$-small geodesic from $v$ to $v^{\prime}$ of length at most $\alpha$ whose initial edge $e^{\prime}$ satisfies $\left(e, e^{\prime}\right) \in \Theta$. Then the cardinality of $V^{\prime}$ is bounded by a number depending only on $\Theta$ and $\alpha$.

Proof. This is an immediate consequence of the first statement in Lemma 2.1 and the cofiniteness of the action of $G$ on the set of edges.

Definition 2.3. We define $\Theta^{(3)}$ as the set of all angles $\left(e, e^{\prime}\right)$ such that there exists a geodesic triangle (possibly with some corners in $\partial \Gamma$ ) with sides $c, c^{\prime}$ and $c^{\prime \prime}$ such that $c$ and $c^{\prime}$ determine the angle $\left(e, e^{\prime}\right)$ at the corner $v \in V$, and such that $c^{\prime \prime}$ does not meet $v$.

Lemma 2.4. The set $\Theta^{(3)}$ is a size for angles.

Proof. Clearly, $G$ acts on $\Theta^{(3)}$ and $\Theta^{(3)}$ contains all trivial angles. To show that $\Theta^{(3)}$ is cofinite it suffices to show by Lemma 2.1, that for all $e$ there are only finitely many $e^{\prime}$ with $\left(e, e^{\prime}\right) \in \Theta^{(3)}$. We will use hyperbolicity of $\Gamma$ to show for each $e^{\prime}$ with $\left(e, e^{\prime}\right) \in \Theta^{(3)}$ there is a circuit of uniformly bounded length in $\Gamma$ that contains both $e$ and $e^{\prime}$. Since $\Gamma$ is fine there are only finitely many such circuits.

To construct the circuit let $c, c^{\prime}$ and $c^{\prime \prime}$ be sides of a geodesic triangle such that $c$ and $c^{\prime}$ determine the angle $\left(e, e^{\prime}\right)$ at the corner $v \in V$, and such that $c^{\prime \prime}$ does not meet $v$. Now pick $w \in c$ as follows. Recall that we picked the hyperbolicity constant $\delta$ to be an integer. If the length of $c$ is $\geq 3 \delta$, then let $w \in c$ with $d_{\Gamma}(v, w)=3 \delta$. Otherwise, let $w$ be the endpoint of $c$ (not $v$ ). Similarly, pick $w^{\prime} \in c^{\prime}$. Hyperbolicity implies that there is a path of length $\leq 10 \delta$ between $w$ and $w^{\prime}$ that misses $v$. Indeed, if $d_{\Gamma}\left(w, c^{\prime}\right) \leq \delta$ then we can connect $w$ first to $c^{\prime}$ and then to $w^{\prime}$ along $c^{\prime}$. If $d_{\Gamma}\left(w^{\prime}, c\right) \leq \delta$ then we can connect $w^{\prime}$ first to $c$ and then to $w$ along $c$. Otherwise, by hyperbolicity, we have $d_{\Gamma}\left(w, c^{\prime \prime}\right) \leq \delta$ and $d_{\Gamma}\left(w^{\prime}, c^{\prime \prime}\right) \leq \delta$ and we can first connect $w$ and $w^{\prime}$ to $c^{\prime \prime}$, and then connect along $c^{\prime \prime}$. Altogether we have constructed a loop of length at most $16 \delta$ that meets $v$ exactly once in the angle $\left(e, e^{\prime}\right)$. The loop may not be embedded, but we can shorten it to produce a circuit containing the angle $\left(e, e^{\prime}\right)$.

Remark 2.5. By cutting geodesic $n$-gons in $\Gamma$ into geodesic triangles one obtains a version of Lemma 2.4 for geodesic $n$-gons. 
For $n \geq 3$ let $(n-2) \Theta^{(3)}$ be the $n-2$-fold sum of $\Theta^{(3)}$. Suppose that the geodesics $c_{1}, \ldots, c_{n}$ are the sides of an $n$-gon in $\Gamma$ (possibly with some corners in $\partial \Gamma)$. Let $\left(e, e^{\prime}\right)$ be the angle in $\Gamma$ determined by the corner $v \in V$ of the $n$-gon between $c_{1}$ and $c_{2}$. If $v \notin c_{3} \cup \cdots \cup c_{n}$ then $\left(e, e^{\prime}\right) \in(n-2) \Theta^{(3)}$.

Similarly, if $v$ is an internal vertex of $c_{i}$ that does not belong to any of the $c_{j}$, $j \neq i$, then $\Varangle_{v} c_{i} \in(n-1) \Theta^{(3)}$.

Lemma 2.6. Let $c$ and $c^{\prime}$ be two geodesics from $v \in V$ to $\xi \in \Delta_{+}, \xi \neq v$. Let $e$ and $e^{\prime}$ be the initial edges of $c$ and $c^{\prime}$. Then $\left(e, e^{\prime}\right)$ is $\Theta^{(3)}$-small.

Proof. If $e \neq e^{\prime}$ then we can subdivide $c$ or $c^{\prime}$ and obtain a geodesic triangle for which $\left(e, e^{\prime}\right)$ will be the angle at $v$ and for which $v$ does not belong to all three sides. Thus $\left(e, e^{\prime}\right) \in \Theta^{(3)}$.

Lemma 2.7. Let $c$ and $c^{\prime}$ be two geodesics between $\xi_{-}$and $\xi_{+} \in \Delta_{+}$. Let $v \in c$ and $v^{\prime} \in c^{\prime}$ be such that no geodesic between $v$ and $v^{\prime}$ is contained in $c \cup c^{\prime}$. Then there exists a $2 \Theta^{(3)}$-small geodesic $\hat{c}$ between $v$ and $v^{\prime}$.

Proof. Choose a geodesic $\hat{c}$ from $v$ to $v^{\prime}$, that first travels along $c$, then along a geodesic $c_{1}$ that meets $c$ and $c^{\prime}$ only in its endpoints and finally along $c^{\prime}$. We set $c_{0}:=c \cap \hat{c}$ and $c_{2}:=c^{\prime} \cap \hat{c}$. Then $c_{0}$ is disjoint from $c^{\prime}$, and $c_{2}$ is disjoint from $c$, since otherwise there is a geodesic from $v$ to $v^{\prime}$ that is contained in $c \cup c^{\prime}$. Now $c_{1}$ splits the bi-gone formed by $c$ and $c^{\prime}$ in two geodesic triangles. For any internal vertex $w$ of $\hat{c}$ we can use one of these two triangles and apply Remark 2.5 to conclude that $\Varangle_{w} \hat{c}$ is $2 \Theta^{(3)}$-small.

Existence of geodesics. The following (presumably well-known) fact is implicitly used in [13, but not explicitly stated.

Lemma 2.8. For $\xi \neq \xi^{\prime} \in \Delta_{+}$, there exists a geodesic between $\xi$ and $\xi^{\prime}$.

Proof. We will use angles to extend the proofs in the locally finite case from [14, Lem. 3.1,3.2, p.428].

First, we consider the case $\xi^{\prime}=v \in V$. If also $\xi \in V$, then the existence of a geodesic between $\xi$ and $v$ is obvious. Let $c$ be a geodesic ray from some vertex $w$ to $\xi$. For $n \in \mathbb{N}$ pick finite geodesics $c_{n}$ from $v$ to $c(n)$. It will be convenient to assume that $c_{n} \cap c$ is a finite geodesic, i.e., $c_{n}$ does not leave $c$ after meeting $c$. We claim, that, as in the locally finite case, a subsequence of $c_{n}$ converges pointwise to a geodesic ray $c^{\prime}$. This ray is then a ray from $v$ to $\xi$. Let $e_{n}$ be the initial edge of $c_{n}$. Then we can use the geodesic triangle whose sides are $c_{n}, c_{m}$ and $c_{[c(n), c(m)]}$ to deduce that $\left(e_{n}, e_{m}\right)$ is $\Theta^{(3)}$-small. It follows that the $e_{n}$ range only over a finite set and we can pick a subsequence $I \subseteq \mathbb{N}$ with $e=e_{n}, n \in I$ constant. Inductively we can now produce a subsequence of the $c_{n}$ that converges pointwise as claimed.

Next, we consider the case $\xi, \xi^{\prime} \in \partial \Gamma$. Let $c$ and $c^{\prime}$ be geodesic rays to $\xi$ and $\xi^{\prime}$. By the first case we can assume $c(0)=c^{\prime}(0)$. We can also assume $c \cap c^{\prime}=c(0)$. Let $c_{n}$ be a finite geodesic from $c(n)$ to $c^{\prime}(n)$. We claim that, as in the locally finite case, there is a vertex $p$ that belongs to infinitely many of the $c_{n}$. Since $\xi \neq \xi^{\prime}$ there is $n_{0} \in \mathbb{N}$ with $d_{\Gamma}\left(c\left(n_{0}\right), c^{\prime}\right)>\delta$. Hyperbolicity implies then $d_{\Gamma}\left(c\left(n_{0}\right), c_{n}\right) \leq \delta$ for $n \geq n_{0}$. We can therefore for $n \geq n_{0}$ pick geodesics $\tilde{c}_{n}$ starting in $c\left(n_{0}\right)$ and ending in $w_{n} \in c_{n}$ of length $\leq \delta$. We can assume $\tilde{c}_{n} \cap c_{n}=w_{n}$. Let $w_{n}^{\prime}$ be the last vertex on $\tilde{c}_{n}$ that is also on $c$. Since $d_{\Gamma}\left(w_{n}^{\prime}, c\left(n_{0}\right)\right) \leq \delta$ and $w_{n}^{\prime} \in c$ there is a subsequence $I \subseteq \mathbb{N}$ with $w^{\prime}=w_{n}^{\prime}, n \in I$ constant. Consider for $n \in I$ now the geodesic triangles whose sides are $\left.c\right|_{\left[w^{\prime}, c(n)\right]},\left.c_{n}\right|_{\left[c(n), w_{n}\right]}$ and $\left.\tilde{c}_{n}\right|_{\left[w^{\prime}, w_{n}\right]}$. Using Remark 2.5 it follows that $\left.\tilde{c}_{n}\right|_{\left[w^{\prime}, w_{n}\right]}$ is $2 \Theta^{(3)}$-small. It also follows that the initial edge $e_{n}$ of $\left.\tilde{c}_{n}\right|_{\left[w^{\prime}, w_{n}\right]}$ forms a $\Theta^{(3)}$-small angle with an edge $e$ of $c$ incident to $w^{\prime}$. Now Corollary 2.2 implies that the $w_{n}$ range only over a finite set. Thus after passing to a further 
subsequence we can assume that there is a vertex $p$ contained in all $c_{n}, n \in I$. Now the argument used in the first case allows us to pass to a further subsequence and to assume that $\left.c_{n}\right|_{[p, c(n)]}, n \in I$ converges pointwise to a geodesic ray from $p$ to $\xi$ and that $\left.c_{n}\right|_{\left[p, c^{\prime}(n)\right]}, n \in I$ converges pointwise to a geodesic ray from $p$ to $\xi^{\prime}$. These two rays now combine to a bi-infinite geodesic between $\xi$ and $\xi^{\prime}$.

The observer topology. Bowditch [13, Sec. 8] defined a topology on $\Delta$; this topology is sometimes called the observer topology. This topology naturally is also defined on $\Delta_{+}=\partial \Gamma \cup V$. We recall a basis for the observer topology. For $\xi \in \Delta_{+}$ and a finite subset $V_{0} \subseteq V$ let $M\left(\xi, V_{0}\right)$ consist of all $\xi^{\prime} \in \Delta_{+}$for which all geodesics between $\xi$ and $\xi^{\prime}$ miss $V_{0} \backslash\{\xi\}$. The $M\left(\xi, V_{0}\right)$ form an open basis for the observer topology. An important fact [13, p. 51] is that the $\forall$ in the definition of $M\left(\xi, V_{0}\right)$ can be replaced with $\exists$ without changing the topology: the sets $M^{\prime}\left(\xi, V_{0}\right)$, defined to consist of all $\xi^{\prime}$ for which there exists a geodesic between $\xi$ and $\xi^{\prime}$ missing $V_{0} \backslash\{\xi\}$, also form an open neighborhood basis for the observer topology. The $M\left(v, V_{0}\right)$ with $v \in V$ and $V_{0} \subset V$ finite are a countable basis for the observer topology, see the discussion preceding Lemma 8.4 in 13 . The observer topology is compact and in particular Hausdorff [13, Lem. 8.4, 8.6]. A convenient procedure to produce convergent subsequences in $\Delta_{+}$is reviewed in Lemma 2.9. The $M\left(v, V_{0}\right)$ with $v \in V$ observer topology has a countable basis, As a compact space with a countable basis for the topology $\Delta_{+}$is metrizable.

Lemma 2.9. Let $\left(\xi_{n}\right)_{n \in \mathbb{N}}$ be a sequence in $\Delta_{+}$. Let $v \in V$. For each $n$, let $c_{n}$ be a geodesic from $v$ to $\xi_{n}$. Then there exists a subsequence $I \subseteq \mathbb{N}$ such that $\xi_{n} \rightarrow \xi \in \Delta_{+}$and moreover the $c_{n}, n \in I$ converge as follows

a) if $\xi \in \partial \Gamma$, then the $c_{n}, n \in I$ converge pointwise to a geodesic ray from $v$ to $\xi$

b) if $\xi \in V$, then $\xi \in c_{n}$ for all $n \in I$ and each edge e incident to $\xi$ is the initial edge for the restriction $\left.c_{n}\right|_{\left[\xi, \xi_{n}\right]}$ for at most finitely many $n \in I$.

Proof. For each $k$ let $c_{n}(k)$ be the $k$-th vertex along $c_{n}$ (starting from $v=c_{n}(0)$ ). If $d_{\Gamma}\left(v, \xi_{n}\right)<k$, then we set $c_{n}(k):=\xi_{n}$.

If $V_{k}:=\left\{c_{n}(k) \mid n \in \mathbb{N}\right\}$ is finite for all $k$ then (using a diagonal subsequence) we can pick a subsequence $I \subseteq \mathbb{N}$ such that $c(k):=c_{n}(k), n \in I$ is eventually constant. If $d_{\Gamma}(v, c(k)) \rightarrow \infty$ with $k \rightarrow \infty, k \in \mathbb{N}$, then the $c_{n}, n \in I$ converge pointwise to a geodesic ray $c$ whose endpoint $\xi$ is also a limit for the $\xi_{n}$. In particular, a) holds. If $d_{\Gamma}(v, c(k)) \nrightarrow \rightarrow \infty$, then for all sufficiently large $n \in I, k \in \mathbb{N}, \xi_{n}=c_{n}(k)=c(k)=$ $\xi \in V$ must be constant. In this case both $\xi_{n}$ and $c_{n}, n \in I$ are eventually constant and b) holds.

If not all $V_{k}$ are finite, then there is $k_{0}$ such that $V_{k}$ is finite for all $k<k_{0}$ and $V_{k_{0}}$ is infinite. Then we find a subsequence $I \subseteq \mathbb{N}$ such that $c_{i}(k)$ is constant in $i$ for $k<k_{0}$ and $c_{i}\left(k_{0}\right) \neq c_{j}\left(k_{0}\right)$ for $i \neq j \in I$. Then $\xi_{i} \rightarrow \xi:=c_{i}\left(k_{0}-1\right) \in V$ for $i \in I, i \rightarrow \infty$ and again $\mathrm{b})$ holds.

Addendum 2.10. Suppose that in Lemma 2.9 in addition $v$ is a vertex of finite valency and that the $c_{n}$ are all $\Theta$-small for some size for angles $\Theta$. Assume also the $\xi_{n}$ eventually leave every ball of finite radius in $\Gamma$. Then $\xi \in \partial \Gamma$ and, for a subsequence, the $c_{n}$ converge pointwise to a geodesic ray from $v$ to $\xi$.

Proof. Corollary 2.2 implies that the $V_{k}$ appearing in the proof of Lemma 2.9] are all finite. Since the $\xi_{n}$ leave eventually every ball of finite radius in $\Gamma$ we have $d_{\Gamma}(v, c(k)) \rightarrow \infty$ with $k \rightarrow \infty$ in the proof of Lemma 2.9 and the result follows.

Large angles. If the angle of a geodesic at some vertex $v$ is large, then this often forces further geodesics to pass through $v$. In the next few lemmas we collect some 
results of this kind. Results of this form are very common in connection with relatively hyperbolic groups, see for example [30, Lem. 3].

Lemma 2.11. Let $c$ be a geodesic between $\xi_{-}$and $\xi_{+}$. Assume that $v$ is an internal vertex of $c$ and that $\Varangle_{v} c$ is $\Theta^{(3)}$-large. Then any other geodesic $c^{\prime}$ between $\xi_{-}$and $\xi_{+}$will also pass through $v$.

Proof. Consider the geodesic triangle whose sides are $c^{\prime},\left.c\right|_{\left[v, \xi_{-}\right]}$and $\left.c\right|_{\left[v, \xi_{+}\right]}$. By definition of $\Theta^{(3)}$ we have $v \in c^{\prime}$.

Lemma 2.12. Let $\xi, \xi_{1}$ and $\xi_{2} \in \Delta_{+}$. Let $c_{1}$ be a geodesic between $\xi$ and $\xi_{1}$ and $c$ be a geodesic between $\xi_{1}$ and $\xi_{2}$. Let $\Theta_{0}$ be a size for angles. Let $v \notin c$ be an internal vertex of $c_{1}$ such that the angle $\Varangle_{v} c_{1}$ is $\Theta_{0}+2 \Theta^{(3)}$-large. Then any geodesic $c_{2}$ between $\xi_{2}$ and $\xi$ contains $v$ as an internal vertex and $\Varangle_{v} c_{2}$ is $\Theta_{0}$-large.

Proof. For the first claim, we subdivide $c_{1}$ at $v$ and obtain a 4-gon whose corners are $v, \xi, \xi_{1}$ and $\xi_{2}$. Since $v \notin c$, we must have $v \in c_{2}$ for otherwise the angle of $c_{1}$ at $v$ would need to be $2 \Theta^{(3)}$-small, see Remark 2.5. Let $e_{1}, e_{1}^{\prime}, e_{2}$ and $e_{2}^{\prime}$ be edges incident to $v$ such that $e_{1}$ points towards $\xi_{1}$ along $c_{1}, e_{1}^{\prime}$ points towards $\xi$ along $c_{1}, e_{2}$ points towards $\xi_{2}$ along $c_{2}$, and $e_{2}^{\prime}$ points towards $\xi$ along $c_{2}$. Thus $\left(e_{1}, e_{1}^{\prime}\right)=\Varangle_{v} c_{1}$ and $\left(e_{2}, e_{2}^{\prime}\right)=\Varangle_{v} c_{2}$. Lemma 2.6 implies that $\left(e_{1}^{\prime}, e_{2}^{\prime}\right)$ is $\Theta^{(3)}$-small. Since $v \notin c$, we can use the geodesic triangle whose sides are $c,\left.c_{1}\right|_{\left[v, \xi_{1}\right]}$ and $\left.c_{2}\right|_{\left[v, \xi_{2}\right]}$ to see that $\left(e_{1}, e_{2}\right)$ is also $\Theta^{(3)}$-small. Since $\left(e_{1}, e_{1}^{\prime}\right)$ is $\Theta_{0}+2 \Theta^{(3)}$-large, this implies that $\left(e_{2}, e_{2}^{\prime}\right)$ is $\Theta_{0}$-large.

Lemma 2.13. Let $\Theta_{0}$ be a size for angles. Let $\xi$, $\xi_{1}$ and $\xi_{2} \in \Delta_{+}$. Let $c$ be a geodesic between $\xi_{1}$ and $\xi_{2}, c_{1}$ be a geodesic between $\xi_{1}$ and $\xi, c_{2}$ be a geodesic between $\xi_{2}$ and $\xi$. Suppose that $v$ is an internal vertex of $c, c_{1}$ and $c_{2}$ and that $\Varangle_{v} c$ is $2 \Theta_{0}+3 \Theta^{(3)}$-large. Then $\Varangle_{v} c_{1}$ or $\Varangle_{v} c_{2}$ is $\Theta_{0}$-large.

Proof. Let $e, e^{\prime}, e_{1}, e_{1}^{\prime}, e_{2}, e_{2}^{\prime}$ be edges incident to $v$ such that $e$ points towards $\xi_{1}$ along $c, e^{\prime}$ points towards $\xi_{2}$ along $c, e_{1}$ points towards $\xi_{1}$ along $c_{1}, e_{1}^{\prime}$ points towards $\xi$ along $c_{1}, e_{2}$ points towards $\xi_{2}$ along $c_{2}$, and $e_{2}^{\prime}$ points towards $\xi$ along $c_{2}$. Thus $\left(e, e^{\prime}\right)=\Varangle_{v} c,\left(e_{1}, e_{1}^{\prime}\right)=\Varangle_{v} c_{1}$, and $\left(e_{2}, e_{2}^{\prime}\right)=\Varangle_{v} c_{2}$. Lemma 2.6 implies that $\left(e_{1}^{\prime}, e_{2}^{\prime}\right)$, $\left(e, e_{1}\right)$ and $\left(e^{\prime}, e_{2}\right)$ are $\Theta^{(3)}$-small. By assumption $\left(e, e^{\prime}\right)$ is $2 \Theta_{0}+3 \Theta^{(3)}$-large. Thus not both $\left(e_{1}, e_{1}^{\prime}\right)$ and $\left(e_{2}, e_{2}^{\prime}\right)$ can be $\Theta_{0}$-small.

Lemma 2.14. Let $\Theta_{0}$ be a size for angles. Then there exists a size $X$ for angles such that the following holds. Assume there is a $\Theta_{0}$-small geodesic c between $\xi_{1}$ and $\xi_{2}$. Let $\xi \in \Delta_{+} \backslash\left\{\xi_{1}, \xi_{2}\right\}$, and $c_{1}$ be a geodesic from $\xi_{1}$ to $\xi$. Let $v \in V$ be an internal vertex of $c_{1}$ for which $\Varangle_{v} c_{1}$ is $X$-large and $v \neq \xi_{2}$. Then any geodesic $c_{2}$ between $\xi_{2}$ and $\xi$ will also pass through $v$. Moreover, for any size for angles $\Theta$ we have

a) if $\Varangle_{v} c_{1}$ is $\Theta$-small, then $\Varangle_{v} c_{2}$ is $\Theta+X$-small;

b) if $\Varangle_{v} c_{1}$ is $\Theta+X$-large, then $\Varangle_{v} c_{2}$ is $\Theta$-large.

Proof. For the first statement we can take $X:=\Theta_{0}+2 \Theta^{(3)}$. Lemma 2.12 implies $v \in c \cup c_{2}$. If $v \notin c_{2}$ then Lemma 2.12 implies that $v$ is an internal vertex of $c$ and that $\Varangle_{v} c$ is $\Theta_{0}$ large. But $c$ is assumed to be $\Theta_{0}$-small and thus $v \in c_{2}$.

For the second statement we can take $X:=\Theta_{0}+3 \Theta^{(3)}$. Let $e_{1}, e_{1}^{\prime}, e_{2}, e_{2}^{\prime}$ be the edges incident to $v$, such that $e_{1}$ points towards $\xi_{1}$ along $c_{1}, e_{1}^{\prime}$ points towards $\xi$ along $c_{1}, e_{2}$ points towards $\xi_{2}$ along $c_{2}$, and $e_{2}^{\prime}$ points towards $\xi$ along $c_{2}$. In particular $\Varangle_{v} c_{1}=\left(e_{1}, e_{1}^{\prime}\right)$ and $\Varangle_{v} c_{2}=\left(e_{2}, e_{2}^{\prime}\right)$. In order to prove a) and $\left.\mathrm{b}\right)$ it will suffice to show that $\left(e_{1}^{\prime}, e_{2}^{\prime}\right)$ is $\Theta^{(3)}$-small and that $\left(e_{1}, e_{2}\right)$ is $\Theta_{0}+2 \Theta^{(3)}$-small. The first statement follows from Lemma 2.6. For the second statement we need to distinguish the cases $v \notin c$ and $v \in c$, i.e., to distinguish whether only $c_{1}$ and $c_{2}$ 
intersect in $v$, or whether all three geodesics intersect in $v$. If $v \notin c$ we can use the geodesic triangle whose sides are $c,\left.c_{1}\right|_{\left[v, \xi_{1}\right]}$ and $\left.c_{2}\right|_{\left[v, \xi_{2}\right]}$ to deduce that $\left(e_{1}, e_{2}\right)$ is $\Theta^{(3)}$-small. If $v \in c$, then let $e$ and $e^{\prime}$ be the edges on $c$ incident to $v$, such that $e$ points towards $\xi_{1}$ along $c$, and $e^{\prime}$ points towards $\xi_{2}$ along $c$. Lemma 2.6 implies that the angles $\left(e, e_{1}\right)$ and $\left(e^{\prime}, e_{2}\right)$ are $\Theta^{(3)}$-small. Since $c$ is $\Theta_{0}$-small the angle $\left(e, e^{\prime}\right)$ is $\Theta_{0}$-small. Thus $\left(e_{1}, e_{2}\right)$ is indeed $\Theta_{0}+2 \Theta^{(3)}$-small.

Lemma 2.15. Let $\xi \in \Delta_{+}$and $v \in V$ where $\xi \neq v$. Then there exist neighborhoods $U$ of $v$ and $U^{\prime}$ of $\xi$ in $\Delta_{+}$such that any geodesic starting in $U$ and ending in $U^{\prime}$ will meet $v$.

Proof. Assume this fails. Then there are $\xi_{n}, x_{n} \in \Delta_{+}$with $\xi_{n} \rightarrow \xi$ and $x_{n} \rightarrow v$ for which there are geodesics $b_{n}$ from $\xi_{n}$ to $x_{n}$ in $\Gamma$ that do not meet $v$. Let $c$ be a geodesic from $\xi$ to $v, a_{n}$ be geodesics from $v$ to $x_{n}$ and $c_{n}$ be geodesics from $\xi_{n}$ to $\xi$. Since $x_{n} \rightarrow v$, the initial edges of the $a_{n}$ form an infinite set. Lemma 2.1 and Remark 2.5 imply that not all angles at $v$ determined by $c$ and $a_{n}$ can be $2 \Theta^{(3)}$ small. On the other hand we can consider the geodesic 4-gon with sides $c, a_{n}, b_{n}$ and $c_{n}$. Since the angle at $v$ determined by $c$ and $a_{n}$ is eventually not $2 \Theta^{(3)}$-small, eventually $v \in b_{n} \cup c_{n}$. Since $\xi_{n} \rightarrow \xi$, we can arrange that the $c_{n}$ eventually miss $v$. This implies that eventually $v \in b_{n}$, contradicting our assumption.

\section{Isotropy groups.}

Lemma 2.16. Let $\xi \in \Delta_{+}, v \in V$ where $v \neq \xi$. There is a neighborhood $U$ of $\xi \in \Delta_{+}$such that only finitely many edges in $\Gamma$ appear as the initial edge of a geodesic from $v$ to some $\xi^{\prime} \in U$.

Proof. Assume this fails. Then there are $\xi_{n} \in \Delta_{+}$, geodesics $c_{n}$ from $v$ to $\xi_{n}$ such that $\xi_{n} \rightarrow \xi$ and the initial edges of the $c_{n}$ are pairwise different. Since the initial edges of the $c_{n}$ are all different it follows that $\xi_{n} \rightarrow v$. But $v \neq \xi$.

Lemma 2.17. Let $\xi_{-} \neq \xi_{+} \in \Delta_{+}$. Then the intersection of the isotropy groups $G_{\xi_{-}} \cap G_{\xi_{+}}$is virtually cyclic.

Proof. If $\xi_{-} \in V$, then, by Lemma 2.16 there are only finitely many edges that are incident to $\xi_{-}$and are part of a geodesic from $\xi_{-}$to $\xi_{+}$. The group $G_{\xi_{-}} \cap G_{\xi_{+}}$acts on this finite set. Since the action of $G$ on the set of all edges is proper, this implies that $G_{\xi_{-}} \cap G_{\xi_{+}}$is finite. The same argument applies if $\xi_{+} \in V$.

It remains to treat the case $\xi_{-}, \xi_{+} \in \partial \Gamma$. Let $L$ be the subgraph of $\Gamma$ spanned by all geodesics from $\xi_{-}$to $\xi_{+}$. By hyperbolicity $L$ is contained in a bounded neighborhood of a fixed geodesic $c$ between $\xi_{-}$and $\xi_{+}$. By [13, Lem. 8.2] the intersection of $L$ with any bounded ball in $\Gamma$ around a vertex $v$ from $L$ contains only finitely many vertices, in particular, $L$ is locally finite.

Since the action of $G$ on edges is proper, the action of $G_{\xi_{-}} \cap G_{\xi_{+}}$on $L$ is proper as well. Fix a vertex $v_{0} \in c$. Now, if the group $G_{\xi_{-}} \cap G_{\xi_{+}}$is infinite, then it contains elements $g$, with $d_{\Gamma}\left(v_{0}, g v_{0}\right)$ arbitrarily large. If $d_{\Gamma}\left(v_{0}, g v_{0}\right)>>0$, then, since $L$ is contained in a $\delta$-neighborhood of the geodesic $c$, it follows that either $d_{\Gamma}\left(v_{0}, g^{2} v_{0}\right) \sim 0$ or $d_{\Gamma}\left(v_{0}, g^{2} v_{0}\right) \sim 2 d_{\Gamma}\left(v_{0}, g v_{0}\right)$. In both cases, again since $L$ is contained in a bounded neighborhood of $c$, we get a good understanding of the action of $g$ on $L$. In the first case $g$ will act, up to bounded error, as reflection with respect to a point of $c$. In particular, $g$ will exchange $\xi_{-}$and $\xi_{+}$. This excludes the first case. In the second cases $g$ acts, up to bounded error, like a translation along $c$. It follows that $g^{ \pm n} v_{0} \rightarrow \xi_{ \pm}$, and, in particular, that the action on $L$ of the cyclic group $C$ generated by $g$ is cocompact. Moreover, since $L$ is contained in a bounded neighborhood of $L$ and since bounded balls in $L$ are finite and since the action of $G$ on the edges of $L$ is proper, it follows that $C$ has finite index in $G_{\xi_{-}} \cap G_{\xi_{+}}$. 
Remark 2.18. Suppose that the group $G_{\xi_{-}} \cap G_{\xi_{+}}$appearing in Lemma 2.17 is infinite virtually cyclic. Then, since $G_{\xi_{-}} \cap G_{\xi_{+}}$fixes the two ends of $L$, this group is virtually cyclic of type I, i.e., admits a surjection to an infinite cyclic group.

\section{Sequences of bounded distance.}

Lemma 2.19. Let $\left(v_{n}\right)_{n \in \mathbb{N}}$ be a sequence in $V$ that converges in $\Delta_{+}$to $\xi \in \partial \Gamma$. If $\left(v_{n}^{\prime}\right)_{n \in \mathbb{N}}$ is another sequence in $V$ such that $d_{\Gamma}\left(v_{n}, v_{n}^{\prime}\right)$ is bounded, then $v_{n}^{\prime}$ also converges to $\xi$.

Proof. Assume this fails. As $\Delta_{+}$is compact there is then a convergent subsequence $\left(v_{n}^{\prime}\right)_{n \in I}$ with $\lim _{n \in I} v_{n}^{\prime}=: \xi^{\prime} \neq \xi$. If $\xi^{\prime} \in \partial \Gamma$, then we can use Lemma 2.9 to produce geodesic rays $c$ to $\xi$ and $c^{\prime}$ to $\xi^{\prime}$ such that, after passing to a further subsequence, we can assume $v_{i} \in c$ and $v_{i}^{\prime} \in c^{\prime}$ for all $i \in I$. Then, by hyperbolicity, $c$ and $c^{\prime}$ are asymptotic and $\xi=\xi^{\prime}$.

It remains to contradict $\xi^{\prime} \in V$. If $\xi^{\prime} \in V$, then Lemma 2.15 allows us to assume, after passing to a further subsequence, that for all $i \in I$ any geodesic between $v_{i}$ and $v_{i}^{\prime}$ passes through $\xi^{\prime}$. But then $d_{\Gamma}\left(\xi^{\prime}, v_{i}\right)$ is bounded and this contradicts $\xi \in \partial \Gamma$.

Addendum 2.20. Retain the assumptions of Lemma 2.19, but assume $\xi=v \in V$. Assume in addition that there is $d>0$ and a size for angles $\Theta$ such that there are $\Theta$-small geodesics $c_{n}$ of length $\leq d$ between $v_{n}$ and $v_{n}^{\prime}$. Assume also that the $v_{n}^{\prime}$ do not converge to $v$. Then there is a subsequence $I \subseteq \mathbb{N}$ such that both $v_{n}$ and $v_{n}^{\prime}$ are constant for $n \in I$.

Proof. We can argue as in the proof of Lemma 2.19 and assume that $\xi^{\prime}=\lim _{n} v_{n}^{\prime}$ exists in $\Delta_{+}$with $\xi^{\prime} \neq v$. Using Lemma 2.15 as in the last paragraph of proof of Lemma 2.19, we can assume that the $c_{n}, n \in I$ all pass through $v$. Lemma 2.16 allows us, after passing to a further subsequence, to assume that the initial edge of the restriction $\left.c_{n}\right|_{\left[v, v_{n}^{\prime}\right]}$ does not depend on $n \in I$. Since the $c_{n}$ are $\Theta$-small, it follows from Lemma 2.1 that the initial edges of the $\left.c_{n}\right|_{\left[v, v_{n}\right]}, n \in I$, can only vary over a finite set of edges. But now, as $v_{n} \rightarrow v$, we must have $v_{n}=v$ for all sufficiently large $n \in I$. Since the $c_{n}$ are $\Theta$-small and of bounded length, Corollary 2.2 implies that the $v_{n}^{\prime}, n \in I$ also vary only over a finite set.

\section{Coarse convexity.}

Lemma 2.21. Let $\xi \in \Delta_{+}$and $U$ be a neighborhood of $\xi \in \Delta_{+}$. Then there exists a neighborhood $U^{\prime}$ of $\xi$ with the following property. If $v_{-}, v_{+} \in U^{\prime}$ and $v$ belongs to a geodesic between $v_{-}$and $v_{+}$then $v \in U$.

Proof. Assume this fails. Then we find sequence of vertices $\left(v_{-}\right)_{n},\left(v_{+}\right)_{n}, v_{n}$ such that $\left(v_{-}\right)_{n} \rightarrow \xi,\left(v_{+}\right)_{n} \rightarrow \xi, v_{n} \notin U$ and $v_{n}$ belongs to a geodesic $c_{n}$ between $\left(v_{-}\right)_{n}$ and $\left(v_{+}\right)_{n}$. If $\xi \in V$, then we apply Lemma 2.15 to $\left.c_{n}\right|_{\left[v_{n},\left(v_{-}\right)_{n}\right]}$ and to $\left.c_{n}\right|_{\left[v_{n},\left(v_{+}\right)_{n}\right]}$. Lemma 2.15 implies that both restrictions eventually contain $\xi$, which can only happen if $v_{n}=\xi$. Since this contradicts $v_{n} \notin U$ we have $\xi \in \partial \Gamma$. Hyperbolicity implies that there are $v_{n}^{\prime}$ such that $v_{n}^{\prime}$ belongs to a geodesic from $\left(v_{-}\right)_{n}$ to $\xi$ or to a geodesic from $\left(v_{+}\right)_{n}$ to $\xi$ and such that $d_{\Gamma}\left(v_{n}, v_{n}^{\prime}\right)$ is uniformly bounded. The first property ensures $v_{n}^{\prime} \rightarrow \xi$ since $\left(v_{-}\right)_{n} \rightarrow \xi$ and $\left(v_{+}\right)_{n} \rightarrow \xi$. The second property allows us to apply Lemma 2.19 and deduce that also $v_{n} \rightarrow \xi$. This contradicts again $v_{n} \notin U$.

\section{The COARSE $\Theta$-FLOW SPACE FOR RELATIVELY HYPERBOLIC GROUPS}

Throughout this section we use the notations and assumptions from Section 2. In particular, $G$ is a group with a simplicial cocompact action on a fine and hyperbolic graph $\Gamma$. The stabilizers of edges under this action are finite. We fix again a 
hyperbolicity constant $\delta \geq 0$ for $\Gamma$. We will use the family $\mathcal{P}$ consisting of all subgroups $H \leq G$ that are virtually cyclic or fix a vertex $v \in V$. The space $\Delta$ is the union $\partial \Gamma \cup V_{\infty}$ and equipped with the observer topology. We also fix a proper left invariant metric $d_{G}$ on $G$.

Theorem 3.1. The action of $G$ on $\Delta$ is finitely $\mathcal{P}$-amenable.

Proof. This will be a consequence of Proposition 3.2 and 3.17.

Covering $G \times{ }_{\Theta_{0}} \partial \Gamma$. It will be convenient to replace $\Gamma$ with its first barycentric subdivision $\Gamma^{\prime}$. The set of vertices in the barycentric subdivision corresponding to the edges of the original graph will be denoted $V_{E}$. Thus the set of vertices of $\Gamma^{\prime}$ is the disjoint union $V \cup V_{E}$, where $V$ is the set of vertices of $\Gamma$. The vertices in $V_{E}$ are all of valence 2 . We will give edges in $\Gamma^{\prime}$ the length $1 / 2$. Then the path length metric of $\Gamma$ and $\Gamma^{\prime}$ coincide. We will in this subsection use $\delta^{\prime}:=\delta+1$. This has the effect that we can use vertices from $V_{E}$ when we apply hyperbolicity: for any geodesic triangle and any vertex $v$ from $V_{E}$ on one side of the triangle there is a vertex $w$ from $V_{E}$ on one of the two other sides with $d_{\Gamma}(v, w) \leq \delta^{\prime}$. Moreover, $\Gamma^{\prime}$ is still fine and the considerations from Section 2 and the appendix all apply to $\Gamma^{\prime}$ as well. In particular, we can define $\Delta^{\prime}$ and $\Delta_{+}^{\prime}$. Since all vertices in $V_{E}$ are of valence 2 we have $\Delta^{\prime}=\Delta$ and $\Delta_{+}^{\prime}=\Delta_{+} \cup V_{E}$. Of course, $\partial \Gamma=\partial \Gamma^{\prime}$. For $v \in V$ there is a canonical bijection between angles at $v$ with respect to $\Gamma$ and angles with respect to $\Gamma^{\prime}$. In this subsection sizes for angles will only be considered at vertices from $V$. (Since there is only one non-trivial angle at any vertex $v \in V_{E}$, angles at $v \in V_{E}$ are not very interesting.)

We fix a base point $v_{0} \in V_{E}$. For a size for angles $\Theta$ we write $G \times_{\Theta} \partial \Gamma$ for the subset of $G \times \partial \Gamma$ consisting of all pairs $(g, \xi) \in G \times \partial \Gamma$ for which there exists a $\Theta$ small geodesic from $g v_{0}$ to $\xi$. The main result of this subsection produces partial covers of $G \times \Delta$.

Proposition 3.2. There is a number $N$ such that for any $\alpha>0$ and any size for angles $\Theta_{0}$ there exists a $G$-invariant collection $\mathcal{U}$ of VCyc-subsets of $G \times \Delta$ such that

a) the order of $\mathcal{U}$ is at most $N$;

b) for every $(g, \xi) \in G \times \times_{\Theta_{0}} \partial \Gamma$ there is $U \in \mathcal{U}$ with $B_{\alpha}(g) \times\{\xi\} \subseteq U$.

To prove Proposition 3.2 we will construct a coarse flow space and use the long and thin covers from Theorem[1.1. For a size for angles $\Theta$, we define the metric $d_{\Theta}$ on $V_{E}$ as follows. For $v, v^{\prime} \in V_{E}$ we set

$$
d_{\Theta}\left(v, v^{\prime}\right):=\min \sum_{i=1}^{n} d_{\Gamma}\left(w_{i-1}, w_{i}\right)
$$

where the minimum is taken over all finite sequences $v=w_{0}, w_{1}, \ldots, w_{n}=v^{\prime}$ of vertices from $V_{E}$ such that there are $\Theta$-small geodesics between $w_{i-1}$ and $w_{i}$ for all $i$. If there is no such sequence, then $d_{\Theta}\left(v, v^{\prime}\right)=\infty$. If there exists a $\Theta$-small geodesic between $v$ and $v^{\prime}$, then $d_{\Theta}\left(v, v^{\prime}\right)=d_{\Gamma}\left(v, v^{\prime}\right)$, but in general $d_{\Theta}\left(v, v^{\prime}\right) \geq d_{\Gamma}\left(v, v^{\prime}\right)$.

Lemma 3.3. The metric $d_{\Theta}$ on $V_{E}$ is proper.

Proof. If $d_{\Theta}\left(v, v^{\prime}\right) \leq n$, then there is a finite sequence $v=w_{0}, w_{1} \ldots, w_{n}=v^{\prime}$ in $V_{E}$ with $d_{\Theta}\left(w_{i-1}, w_{i}\right) \leq 1$ for all $i$. Therefore, it suffices to check that balls of radius 1 are finite. This is a consequence of Lemma 2.1

Definition 3.4. Set $Z:=\left(\Delta_{+}^{\prime}\right)^{2}$. Let $\Theta$ be a size for angles. The coarse $\Theta$-flow space $C F(\Theta)$ for $\Gamma$ is the subset of $V_{E} \times Z$ consisting of all triples $\left(v, \xi_{-}, \xi_{+}\right)$for which there exist $v^{\prime} \in V_{E}$ and a $\Theta$-small geodesic $c$ between $\xi_{-}$and $\xi_{+}$such that $v^{\prime} \in c$ and $d_{\Theta}\left(v, v^{\prime}\right) \leq \delta^{\prime}$. Moreover, we require $\xi_{-}, \xi_{+} \in V_{E} \cup \partial \Gamma$. 
Example 3.5. Suppose that $\Gamma$ is a locally finite tree. The flow space FS from 4 for $\Gamma$ consists of all generalized (parametrized) geodesics $c: \mathbb{R} \rightarrow \Gamma$. If we use $\delta^{\prime}=0$ and all angles for $\Theta$, then there is a natural embedding $C F(\Theta) \rightarrow F S$ that sends $\left(v, \xi_{-}, \xi_{+}\right)$to the generalized geodesic $c: \mathbb{R} \rightarrow \Gamma$ with $c(-\infty)=\xi_{-}, c(0)=v$ and $c(+\infty)=\xi_{+}$.

Lemma 3.6. Let $\Theta$ be a size for angles with $2 \Theta^{(3)} \subseteq \Theta$. Then

a) $C F(\Theta) \subseteq V_{E} \times Z$ is closed;

b) $\operatorname{dim} C F(\Theta) \leq \operatorname{dim} Z<\infty$;

c) for $\left(\xi_{-}, \xi_{+}\right) \in Z, V_{\xi_{-}, \xi_{+}}:=\left\{v \in V_{E} \mid\left(v, \xi_{-}, \xi_{+}\right) \in C F(\Theta)\right\}$ has the $(D, R)$ doubling property with respect to $d_{\Theta}$, where $D$ and $R$ are independent of $\left(\xi_{-}, \xi_{+}\right)$and $\Theta$

d) for $\left(v, \xi_{-}, \xi_{+}\right)$the isotropy group $G_{\xi_{-}, \xi_{+}}=\left\{g \in G \mid g \xi_{-}=\xi_{-}, g \xi_{+}=\xi_{+}\right\}$is virtually cyclic.

Proof. a) It suffices to show that $Z_{v}:=\left\{\left(\xi_{-}, \xi_{+}\right) \mid\left(v, \xi_{-}, \xi_{+}\right) \in C F(\Theta)\right\}$ is closed for each $v \in V_{E}$. Let $\left(\left(\xi_{-}\right)_{n},\left(\xi_{+}\right)_{n}\right) \in Z_{v}$ with $\left(\left(\xi_{-}\right)_{n},\left(\xi_{+}\right)_{n}\right) \rightarrow\left(\xi_{-}, \xi_{+}\right)$in $Z$. We need to show $\left(\xi_{-}, \xi_{+}\right) \in Z_{v}$. Since $\left(\left(\xi_{-}\right)_{n},\left(\xi_{+}\right)_{n}\right) \in Z_{v}$ there are $\Theta$-small geodesics $c_{n}$ from $\left(\xi_{-}\right)_{n}$ to $\left(\xi_{+}\right)_{n}$ and vertices $v_{n} \in c_{n} \cap V_{E}$ with $d_{\Theta}\left(v_{n}, v\right) \leq \delta^{\prime}$. As $d_{\Theta}$ is proper by Lemma 3.3 we can pass to a subsequence and assume that $v_{n}=w$ is constant. Moreover, we can apply Lemma 2.9 to the $\left.c_{n}\right|_{\left[w,\left(\xi_{-}\right)_{n}\right]}$ and assume that either the $\left.c_{n}\right|_{\left[w,\left(\xi_{-}\right)_{n}\right]}$ converge pointwise to a geodesic ray from $w$ to $\xi_{-} \in \partial \Gamma$, or that $\left.\xi_{-} \in c_{n}\right|_{\left[w,\left(\xi_{-}\right)_{n}\right]}$ for all $n$. In the second case, it also follows that eventually $\left(\xi_{-}\right)_{n}=\xi_{-}$, as otherwise $\left(\xi_{-}\right)_{n} \rightarrow \xi_{-}$would imply that the $\Varangle_{\xi_{-}} c_{n}$ are eventually $\Theta$-large by Lemma 2.1. Therefore, $\xi_{-} \in V_{E} \cup \partial \Gamma$ and the $\left.c_{n}\right|_{\left[w,\left(\xi_{-}\right)_{n}\right]}$ converge pointwise to a geodesic $c_{-}$from $w$ to $\xi_{-}$. Similar, $\xi_{+} \in V_{E} \cup \partial \Gamma$ and we can assume that the $\left.c_{n}\right|_{\left[w,\left(\xi_{+}\right)_{n}\right]}$ converge pointwise to a geodesic $c_{+}$from $w$ to $\xi_{+}$. Now $c_{-}$ and $c_{+}$combine to a geodesic between $\xi_{-}$and $\xi_{+}$that passes through $w$. Thus $\left(\xi_{-}, \xi_{+}\right) \in Z_{v}$.

b) Theorem A.1 asserts that $\Delta_{+}^{\prime}$ is finite dimensional. Since $V_{E}$ is discrete and $C F(\Theta)$ is closed in $V_{E} \times Z$ it follows that $\operatorname{dim} C F(\Theta) \leq \operatorname{dim} Z=2 \operatorname{dim} \Delta_{+}^{\prime}<\infty$.

c) If $V_{\xi_{-}, \xi_{+}}$is non-empty, then there is a $\Theta$-small geodesic $c$ between $\xi_{-}$and $\xi_{+}$. By hyperbolicity, any vertex $v^{\prime} \in V_{E}$ on any other $\Theta$-small geodesic $c^{\prime}$ from $\xi_{-}$to $\xi_{+}$will be within distance $\delta^{\prime}$ of some vertex $v \in V_{E} \cap c$. If $c \cup c^{\prime}$ does not contain a geodesic between $v$ and $v^{\prime}$, then Lemma 2.7 provides us with a $\Theta$-small geodesic between $v$ and $v^{\prime}$. If $c \cup c^{\prime}$ contains a geodesic $c^{\prime \prime}$ between $v$ and $v^{\prime}$, then we can assume that $c^{\prime \prime}$ changes only once from $c$ to $c^{\prime}$, at some vertex $w \in V$. Then $c^{\prime \prime}$ may fail to be $\Theta$-small only at this vertex $w$. In this case we may assume that $v$ is incident to $w$. Let now $\tilde{v} \in V_{E}$ be the unique vertex incident to $w$ on $c^{\prime} \backslash c^{\prime \prime}$. Then $\left.c^{\prime}\right|_{\left[v^{\prime}, \tilde{v}\right]}$ is a $\Theta$-small geodesic of length at most $\delta^{\prime}$ and thus $d_{\Theta}\left(v^{\prime}, \tilde{v}\right) \leq \delta^{\prime}$. We can apply Lemma 2.6 to the bi-gone spanned by $\left.c\right|_{\left[w, \xi_{ \pm}\right]}$and $\left.c^{\prime}\right|_{\left[w, \xi_{ \pm}\right]}$to deduce that the angle at $w$ spanned by the edges $(w, \tilde{v})$ and $(w, v)$ is $\Theta^{(3)}$-small. Thus $d_{\Theta}(v, \tilde{v})=1$ and $d_{\Theta}\left(v, v^{\prime}\right) \leq \delta^{\prime}+1$.

Thus any $v \in V_{\xi_{-}, \xi_{+}}$will be within distance $2 \delta^{\prime}+1$ of a vertex from $V_{E} \cap c$ with respect to $d_{\Theta}$. Therefore, any $\alpha$-separated subset $S$ in a $2 \alpha$-ball in $V_{\xi_{-}, \xi_{+}}$can be mapped injectively to an $\alpha-4 \delta^{\prime}-2$-separated subset $S^{\prime}$ of an $2 \alpha+2 \delta^{\prime}+1$-ball in $V_{E} \cap c$. For sufficiently large $\alpha$ (for example, $\alpha>R:=24 \delta^{\prime}+12$ ) any such set $S^{\prime}$ contains at most 5 elements, since $V_{E} \cap c$ is isometric to a subset of $\mathbb{Z}$.

d) If $\xi_{-} \in V_{E}$ or $\xi_{+} \in V_{E}$, then $G_{\xi_{-}, \xi_{+}}$is finite since the action of $G$ on $V_{E}$ is proper. If $\xi_{-}, \xi_{+} \in \partial \Gamma$, then, by definition of $C F(\Theta), \xi_{-} \neq \xi_{+}$. Lemma 2.17implies that then $G_{\xi_{-}, \xi_{+}}$is virtually cyclic. 
Proposition 3.7. There is a number $N^{\prime}$ such that for any $\alpha^{\prime}>0$ and any size for angles $\Theta$ containing $2 \Theta^{(3)}$ there exists a VCyc-cover $\mathcal{W}$ of $C F(\Theta)$ such that

a) $\operatorname{dim} \mathcal{W} \leq N^{\prime}$

b) for any $\left(v, \xi_{-}, \xi_{+}\right) \in C F(\Theta)$ there is $W \in \mathcal{W}$ such that $B_{\alpha^{\prime}}^{\Theta}(v) \times\left\{\left(\xi_{-}, \xi_{+}\right)\right\} \cap$ $C F(\Theta) \subseteq W$. Here $B_{\alpha^{\prime}}^{\Theta}(v)$ is the $\alpha^{\prime}$-ball in $V_{E}$ with respect to $d_{\Theta}$.

Proof. Let $\Theta$ be a size for angles. Lemma 3.3 and 3.6]allow us to apply Theorem[1.1. We obtain a number $N^{\prime}$ such that for any $\alpha^{\prime}>0$ there exists a VCyc-cover $\mathcal{W}$ of $C F(\Theta)$ satisfying a) and b)

The number $N^{\prime}$ only depends on $\operatorname{dim} C F(\Theta)$ and the doubling constant. For these numbers Lemma 3.6 provides bounds that do not depend on $\Theta$. Therefore $N^{\prime}$ does not depend on $\Theta$.

Lemma 3.8. Let $\alpha>0$ and $\Theta_{0}$ be a size for angles. Then there is a size for angles $\Theta$ containing $\Theta_{0}+2 \Theta^{(3)}$ with the following property: If $c$ is a $\Theta_{0}$-small geodesic from $g v_{0}$ to $\xi \in \partial \Gamma$ and $g^{\prime} \in G$ with $d_{G}\left(g, g^{\prime}\right) \leq \alpha$, then

a) any geodesic $c^{\prime}$ from $g^{\prime} v_{0}$ to $\xi$ is $\Theta$-small;

b) any geodesic $c^{\prime \prime}$ starting on $c$ and ending on a geodesic $c^{\prime}$ from $g^{\prime} v_{0}$ to $\xi$ is $\Theta$-small.

Proof. Pick $\Theta_{1}$ such that for any $h \in B_{\alpha}(e)$ there exists a $\Theta_{1}$-small geodesic from $h v_{0}$ to $v_{0}$. Thus, if $g^{\prime} \in G$ with $d_{G}\left(g, g^{\prime}\right) \leq \alpha$ then there is a $\Theta_{1}$-small geodesic between $g v_{0}$ and $g^{\prime} v_{0}$. Let $X$ be the size for angles from Lemma 2.14. In particular, for any size for angles $Y$, and any geodesic triangle in $\Gamma$ where the first side is $\Theta_{0^{-}}$ small and the second side is $Y$-small, the third side will be $Y+X$-small. Then a) holds whenever $\Theta_{1}+X \subseteq \Theta$ and b) holds whenever $\Theta_{1}+2 X \subseteq \Theta$.

Definition 3.9. Let $W \subseteq C F(\Theta)$ and $\tau \in \mathbb{N}$. We define

$$
\iota^{-\tau} W \subseteq G \times{ }_{\Theta} \partial \Gamma
$$

as the subspace consisting of pairs $(g, \xi) \in G \times{ }_{\Theta} \partial \Gamma$ with the following property. For every $\Theta$-small geodesic $c$ from $g v_{0}$ to $\xi$ we have $\left(v_{c}, g v_{0}, \xi\right) \in W$, where $v_{c}$ is the unique vertex in $V_{E} \cap c$ with $d_{\Gamma}\left(g v_{0}, v_{c}\right)=\tau$.

Lemma 3.10. If $W$ is open in $C F(\Theta)$, then $\iota^{-\tau} W$ is open in $G \times{ }_{\Theta} \partial \Gamma$.

Proof. Let $(g, \xi) \in \iota^{-\tau} W$. Assume that $(g, \xi)$ does not belong to the interior of $\iota^{-\tau} W$ in $G \times{ }_{\Theta} \partial \Gamma$. Then there are $\xi_{n} \in \partial \Gamma$ with $\xi_{n} \rightarrow \xi$ and $\Theta$-small geodesics $c_{n}$ from $g v_{0}$ to $\xi_{n}$ such that for the vertices $v_{n} \in V_{E} \cap c_{n}$ with $d_{\Gamma}\left(g v_{0}, v_{n}\right)=\tau$ we have $\left(v_{n}, g v_{0}, \xi_{n}\right) \notin W$. Using Lemma 2.9 a) and passing to a subsequence we may assume that the $c_{n}$ converge pointwise to a $\Theta$-small geodesic $c$ from $g v_{0}$ to $\xi$. Then eventually $v_{n}=v_{c}$ is constant and belongs to $c$. Since $(g, \xi) \in W$, it follows that $\left(v_{c}, g v_{0}, \xi\right) \in W$. Since $W$ is open, eventually $\left(v_{n}, g v_{0}, \xi_{n}\right) \in W$, contradicting our assumption.

Proof of Proposition 3.2. Let $\Theta_{0}$ and $\alpha>0$ be given. Let $\Theta$ be the size for angles from Lemma 3.8 Since $B_{\alpha}(e) \subseteq G$ is finite, we can find a number $\alpha^{\prime}>0$ such that $d_{\Gamma}\left(g v_{0}, g h v_{0}\right)+2 \delta^{\prime} \leq \alpha^{\prime}$ for all $g \in G$ and $h \in B_{\alpha}(e)$. Let $\mathcal{W}$ be the cover of $C F(\Theta)$ from Proposition 3.7. For $\tau \in \mathbb{N}$ let $\iota^{-\tau} \mathcal{W}:=\left\{\iota^{-\tau} W \mid W \in \mathcal{W}\right\}$. By Lemma 3.10, the members of $\iota^{-\tau} \mathcal{W}$ are open subsets of $G \times{ }_{\Theta} \partial \Gamma$. Since $\iota^{-\tau}$ is a $G$-equivariant operation and $\mathcal{W}$ is $G$-invariant and consist of VCyc-subset, the same is true for $\iota^{-\tau} \mathcal{W}$. The order of $\mathcal{W}$ is bounded by Proposition 3.7 a). Since $\iota^{-\tau}$ commutes with intersection, the order of $\iota^{-\tau} \mathcal{W}$ is bounded by the order of $\mathcal{W}$.

We claim that there exists $\tau \in \mathbb{N}$ such that $\iota^{-\tau} \mathcal{W}$ is $\alpha$-wide in the $G$-direction, i.e., we claim that there is $\tau$ such that

(3.11) for any $(g, \xi) \in G \times \times_{\Theta_{0}} \partial \Gamma$ there is $W \in \mathcal{W}$ with $B_{\alpha}(g) \times\{\xi\} \subseteq \iota^{-\tau} W$. 
Suppose there is no such $\tau$. Then there is a sequence of pairs $\left(g_{\tau}, \xi_{\tau}\right)_{\tau \in \mathbb{N}}$ in $G \times{ }_{\Theta_{0}} \partial \Gamma$ such that

(3.12) for all $W \in \mathcal{W}$ and all $\tau \in \mathbb{N}$ we have $B_{\alpha}\left(g_{\tau}\right) \times\left\{\xi_{\tau}\right\} \nsubseteq \iota^{-\tau} W$.

By definition of $G \times \Theta_{\Theta_{0}} \partial \Gamma$ there are $\Theta_{0}$-small geodesics $c_{\tau}$ from $g_{\tau} v_{0}$ to $\xi_{\tau}$ in $\Gamma$. Let $v_{\tau}$ be the unique vertex on $c_{\tau}$ with $d_{\Gamma}\left(g_{\tau} v_{0}, v_{\tau}\right)=\tau$. Since the action of $G$ on $V$ is cofinite, and since the $\iota^{-\tau} \mathcal{W}$ are $G$-invariant we may, after passing to a subsequence $\left(g_{\tau}, \xi_{\tau}\right)_{\tau \in T}$, assume that $v_{\tau}=v$ is constant. Using the compactness of $\Delta_{+}^{\prime}$, we may, after passing to a further subsequence, assume that $\xi_{-}:=\lim g_{\tau} v_{0}$ and $\xi_{+}:=\lim \xi_{\tau}$ exist in $\Delta_{+}^{\prime}$. Addendum 2.10 implies $\xi_{-}, \xi_{+} \in \partial \Gamma$ and allows us to assume that the restrictions $\left.c_{\tau}\right|_{\left[v, g_{\tau} v_{0}\right]}$ and $\left.c_{\tau}\right|_{\left[v, \xi_{\tau}\right]}$ converge pointwise to geodesic rays $c_{-}$from $v$ to $\xi_{-}$and $c_{+}$from $v$ to $\xi_{+}$. In particular, $c_{-}$can be combined with $c_{+}$to obtain a geodesic $c$ between $\xi_{-}$and $\xi_{+}$. This geodesic $c$ is $\Theta_{0}$-small, since the $c_{\tau}$ are $\Theta_{0}$-small.

By Proposition 3.7] b) there is $W \in \mathcal{W}$ such that $B_{\alpha^{\prime}}^{\Theta}(v) \times\left\{\left(\xi_{-}, \xi_{+}\right)\right\} \cap C F(\Theta) \subseteq$ $W$. Let $V^{\prime}$ be the set of $v^{\prime} \in B_{\alpha^{\prime}}^{\Theta}(v)$ with $d_{\Theta}\left(v^{\prime}, w\right) \leq \delta^{\prime}$ for some $w \in c$. Then $\left(v^{\prime}, \xi_{-}, \xi_{+}\right) \in C F(\Theta)$ for all $v^{\prime} \in V^{\prime}$. Since $d_{\Theta}$ is proper by Lemma 3.3, the set $V^{\prime}$ is finite. Therefore we find neighborhoods $U_{-}$of $\xi_{-}$and $U_{+}$of $\xi_{+}$, such that $V^{\prime} \times U_{-} \times U_{+} \cap C F(\Theta) \subseteq W$. Elements of $B_{\alpha}\left(g_{\tau}\right)$ can be written as $g_{\tau} h$ with $h \in$ $B_{\alpha}(e)$. For each $h, \lim _{\tau} g_{\tau} h v_{0}=\lim _{\tau} g_{\tau} v_{0}=\xi_{-}$by Lemma 2.19. Since $B_{\alpha}(e)$ is finite we find $\tau_{0}$ such that $g_{\tau_{0}} h v_{0} \in U_{-}$for all $h \in B_{\alpha}(e)$. Moreover, we can arrange for $\tau_{0}$ to satisfy $\tau_{0}>\alpha^{\prime}, \xi_{\tau_{0}} \in U_{+}$, and $c_{\tau_{0}} \cap B_{\alpha^{\prime}}(v)=c \cap B_{\alpha^{\prime}}(v)$. We claim that

(3.13) $B_{\alpha}\left(g_{\tau_{0}}\right) \times\left\{\xi_{\tau_{0}}\right\} \subseteq \iota^{-\tau_{0}} W$.

Let $h \in B_{\alpha}(e)$. By Lemma 3.8 a) there is a $\Theta$-small geodesic $c^{\prime}$ from $g_{\tau_{0}} h v_{0}$ to $\xi_{\tau_{0}}$. Let $v^{\prime} \in V_{E} \cap c^{\prime}$ be the vertex with $d_{\Gamma}\left(g_{\tau_{0}} h v_{0}, v^{\prime}\right)=\tau_{0}$. We need to show that $\left(v^{\prime}, g_{\tau_{0}} h v_{0}, \xi_{\tau_{0}}\right)$ belongs to $W$. To this end it suffices to show $v^{\prime} \in V^{\prime}$, where $V^{\prime}$ is as defined earlier. Now we use hyperbolicity to find $w \in V_{E} \cap c_{\tau_{0}}$ with $d_{\Gamma}\left(w, v^{\prime}\right) \leq \delta^{\prime}$. Lemma $\left.3.8 \mathrm{~b}\right)$ implies that there is a $\Theta$-small geodesic between $w$ and $v^{\prime}$. Therefore $d_{\Theta}\left(w, v^{\prime}\right) \leq \delta^{\prime}$. Since $d_{\Gamma}\left(g_{\tau_{0}} v_{0}, g_{\tau_{0}} h v_{0}\right) \leq \alpha^{\prime}-2 \delta^{\prime}$ and $d_{\Gamma}\left(w, v^{\prime}\right) \leq \delta^{\prime}$ we have $d_{\Gamma}\left(g_{\tau_{0}} h v_{0}, v^{\prime}\right)-\left(\alpha^{\prime}-\delta^{\prime}\right) \leq d_{\Gamma}\left(g_{\tau_{0}} v_{0}, w\right) \leq d_{\Gamma}\left(g_{\tau_{0}} h v_{0}, v^{\prime}\right)+\left(\alpha^{\prime}-\delta^{\prime}\right)$. Since $d_{\Gamma}\left(g_{\tau_{0}} v_{0}, v\right)=d_{\Gamma}\left(g_{\tau_{0}} h v_{0}, v^{\prime}\right)=\tau_{0}$ we have $d_{\Gamma}(v, w) \leq \alpha^{\prime}-\delta^{\prime}$. Now $c_{\tau_{0}} \cap B_{\alpha^{\prime}}(v)=$ $c \cap B_{\alpha^{\prime}}(v)$ implies $w \in c$. Since $c$ is $\Theta_{0}$-small and therefore $\Theta$-small, and since $v, w \in c$, we have $d_{\Theta}(w, v) \leq \alpha^{\prime}-\delta^{\prime}$. Therefore $d_{\Theta}\left(v^{\prime}, v\right) \leq \alpha^{\prime}$ and $v^{\prime} \in B_{\alpha^{\prime}}^{\Theta}(v)$. Altogether we have shown $v^{\prime} \in V^{\prime}$ and therefore (3.13). This contradicts (3.12) and finishes the proof of (3.11).

It remains to extend $\iota^{-\tau} \mathcal{W}$ to a $G$-invariant collection of VCyc-subset of $G \times \Delta$ of the same order. Since $\Delta$ is metrizable, there is a $G$-invariant metric on $G \times \Delta$. Thus the needed extension exists by Lemma B.2.

Covering $G \times \Delta \backslash G \times{ }_{\Theta} \partial \Gamma$.

Definition 3.14. Let $v \in V$ and let $\Theta$ be a size for angles in $\Gamma$. Define

$$
V_{+}(v, \Theta) \subseteq G \times \Delta
$$

as the set of all $(g, \xi)$ with the following properties

a) all geodesics from $g v_{0}$ to $v$ are $\Theta$-small;

b) if $\xi \neq v$, then there is a geodesic $c$ in $\Gamma$ from $g v_{0}$ to $\xi$ such that $\Varangle_{v} c$ is $\Theta$-large. (In particular, we require $v \in c$.)

Let $V(v, \Theta)$ be the interior of $V_{+}(v, \Theta)$.

Lemma 3.15. Assume that $\Theta$ is a size for angles containing $\Theta^{(3)}$.

a) For $v \in V$ we have $V_{+}(v, \Theta) \cap G \times V_{\infty} \subseteq V(v, \Theta)$;

b) for $v \neq v^{\prime} \in V$ we have $V(v, \Theta) \cap V\left(v^{\prime}, \Theta\right)=\emptyset$; 
c) if $g V(v, \Theta) \cap V(v, \Theta) \neq \emptyset$ with $g \in G, v \in V$, then $g v=v$ and $g V(v, \Theta)=$ $V(v, \Theta)$.

Proof. a) Let $(g, w) \in V_{+}(v, \Theta)$ with $w \in V_{\infty}$. Let $c$ be a geodesic from $g v_{0}$ to $w$. We proceed by contradiction and assume that $(g, w)$ does not belong to the interior of $V_{+}(v, \Theta)$. Then there is a sequence $\xi_{n}$ in $\Delta$ with $\xi_{n} \rightarrow w$ and $\left(g, \xi_{n}\right) \notin V_{+}(v, \Theta)$. Let $c_{n}$ be a geodesic from $g v_{0}$ to $\xi_{n}$. By Lemma 2.15 the $c_{n}$ will eventually pass through $w$. In this case we can change $c_{n}$ and arrange for $\left.c_{n}\right|_{\left[g v_{0}, w\right]}=c$. If $w \neq v$ then these $c_{n}$ prove that eventually $\left(g, \xi_{n}\right) \in V_{+}(v, \Theta)$ which contradicts our assumption. If $w=v$, then it remains to show that $\Varangle_{v} c_{n}$ will eventually be $\Theta$-large. In this case the initial edges of the restriction $\left.c_{n}\right|_{\left[v, \xi_{n}\right]}$ will form an infinite set since $\xi_{n} \rightarrow v$. This implies, by Lemma 2.1 that $\Varangle_{v} c_{n}$ at $v$ will eventually be $\Theta$-large.

b) Assume there is $(g, \xi) \in V(v, \Theta) \cap V\left(v^{\prime}, \Theta\right)$. Let $c$ and $c^{\prime}$ be geodesics from $g v_{0}$ to $\xi$ such that $\Varangle_{v} c$ is $\Theta$-large (if $v \neq \xi$ ) and $\Varangle_{v^{\prime}} c^{\prime}$ is $\Theta$-large (if $v^{\prime} \neq \xi$ ). Since $\Theta^{(3)} \subseteq \Theta$, Lemma 2.11 implies $v \in c^{\prime}$ and $v^{\prime} \in c$. Assume $v \neq \xi$. Without loss of generality, we may assume that $v$ is closer to $g v_{0}$ than $v^{\prime}$. Then $v$ is an internal vertex of $\left.c\right|_{\left[g v_{0}, v^{\prime}\right]}$. Since $\Varangle_{v} c$ is $\Theta$-large, $\left.c\right|_{\left[g v_{0}, v^{\prime}\right]}$ is not $\Theta$-small. This contradicts $\left(g v_{0}, \xi\right) \in V\left(v^{\prime}, \Theta\right)$.

c) Clearly, $g V(v, \Theta)=V(g v, \Theta)$. Thus b) shows that $g V(v, \Theta) \cap V(v, \Theta) \neq \emptyset$ implies $g v=v$.

Lemma 3.16. Let $\Theta$ be a size for angles. Let $v \in V, g \in G, \xi \in \Delta$. Assume that any geodesic from $g v_{0}$ to $v$ is $\Theta$-small. Assume that there is a geodesic $c$ from $g v_{0}$ to $\xi$ that passes through $v$ and satisfies one of the following two conditions

a) $\Varangle_{v}$ c is $\Theta+2 \Theta^{(3)}$-large;

b) $\Varangle_{v} c$ is $\Theta$-large and there is an interior vertex $w$ of $c$ with $d_{\Gamma}\left(g v_{0}, v\right)<$ $d_{\Gamma}\left(g v_{0}, w\right)$ such that $\Varangle_{w} c$ is $2 \Theta^{(3)}$-large.

Then $(g, \xi) \in V(v, \Theta)$.

Proof. If a) holds, then set $A:=\{v\}$; if b) holds, then set $A:=\{w\}$. Recall that $M^{\prime}(\xi, A)$ consists of all $\xi^{\prime}$ for which there exists a geodesic between $\xi$ and $\xi^{\prime}$ that misses $A \backslash\{\xi\}$. Since $M^{\prime}(\xi, A)$ is part of a neighborhood basis for the observer topology, it suffices to show that $\left(g, \xi^{\prime}\right) \in V_{+}(v, \Theta)$ for all $\xi^{\prime} \in M^{\prime}(\xi, A)$. Let $\xi^{\prime} \in M^{\prime}(\xi, A)$. Let $c^{\prime \prime}$ be a geodesic between $\xi$ and $\xi^{\prime}$ that misses $A$. Let $c^{\prime}$ be a geodesic between $g v_{0}$ and $\xi^{\prime}$. We apply Lemma 2.12 to the geodesic triangle with sides $c, c^{\prime}$ and $c^{\prime \prime}$.

If a) holds, then Lemma 2.12 implies that $c^{\prime}$ passes through $v$ and that $\Varangle_{v} c^{\prime}$ is $\Theta$-large. Thus in this case $\left(g, \xi^{\prime}\right) \in V_{+}(v, \Theta)$.

If b) holds, then Lemma 2.12 implies $w \in c^{\prime}$. Then we can replace $c^{\prime}$ and assume $\left.c^{\prime}\right|_{\left[g v_{0}, w\right]}=\left.c\right|_{\left[g v_{0}, w\right]}$. In particular $\Varangle_{v} c^{\prime}=\Varangle_{v} c$ is $\Theta$-large. Thus in this case $\left(g, \xi^{\prime}\right) \in V_{+}(v, \Theta)$ as well.

Proposition 3.17. Let $\alpha>0$. There is a size for angles $\Theta$ and a $G$-invariant collection $\mathcal{V}$ of $\mathcal{P}$-subsets of $G \times \Delta$ such that

a) the order of $\mathcal{V}$ is at most 2 ;

b) for every $(g, \xi) \in G \times \Delta$ at least one of the following two statements holds

- there is $V \in \mathcal{V}$ such that $B_{\alpha}(g) \times\{\xi\} \subseteq V$;

$-\xi \in \partial \Gamma$ and there is a $\Theta$-small geodesic from $g v_{0}$ to $\xi$.

Proof. Since $\Gamma$ is fine there are only finitely many geodesics between any two vertices. In particular, we can pick a size for angles $\Theta_{0}$ such that for all $h, h^{\prime}, h^{\prime \prime} \in$ $B_{\alpha}(e)$ any geodesic starting at $h v_{0}$ and ending in some vertex on another geodesic between vertices $h^{\prime} v_{0}$ and $h^{\prime \prime} v_{0}$ is $\Theta_{0}$-small. Let $X$ be the size for angles appearing in Lemma 2.14. After increasing $X$, if necessary, we may assume $\Theta_{0}+2 \Theta^{(3)} \subseteq X$. Let 
$\mathcal{V}_{1}:=\{V(v, 2 X) \mid v \in V\}, \mathcal{V}_{2}:=\{V(v, 5 X) \mid v \in V\}$ and $\mathcal{V}_{3}:=\{V(v, 6 X) \mid v \in V\}$ All three collections are $G$-invariant. Lemma 3.15 implies that all three collections are of order 0 , and consist of $\mathcal{P}$-sets. Thus $\mathcal{V}:=\mathcal{V}_{1} \cup \mathcal{V}_{2} \cup \mathcal{V}_{3}$ is a $G$-invariant collection of $\mathcal{P}$-sets and satisfies a)

It remains to check that $\mathcal{V}$ also satisfies b) where we use $\Theta:=6 X$. Since $\mathcal{V}$ is $G$-invariant it suffices to consider $(e, \xi) \in G \times \Delta$.

Let $\mathcal{C}$ be the set of all geodesics from some $h v_{0}, h \in B_{\alpha}(e)$ to $\xi$. If all $c \in \mathcal{C}$ are $6 X$-small, then b) holds. Indeed, if $\xi \in V$, then, using Lemma 3.15 a) we obtain $B_{\alpha}(e) \times\{\xi\} \subseteq V(\xi, 6 X)$ and $\left.\mathrm{b}\right)$ holds. If $\xi \in \partial \Gamma$, then $\left.\mathrm{b}\right)$ holds as well, simply since $\mathcal{C}$ contains a $\Theta$-small geodesic from $v_{0}$ to $\xi$. Therefore we may assume that not all $c \in \mathcal{C}$ are $6 X$-small.

Let $c_{0}$ be a geodesic from $v_{0}$ to $\xi$. Let $W$ be the set of all internal vertices $w$ of $c_{0}$ for which $\Varangle_{w} c_{0}$ is $X$-large. Lemma 2.14 implies for any $c \in \mathcal{C}$ and any size for angles $\Theta^{\prime}$ the following holds:

(3.18) $c$ will pass through any $w \in W$;

(3.19) if $v$ is an internal vertex of $c$, where $v \notin W$, then the angle of $c$ at $v$ is $2 X$-small;

(3.20) if, for $w \in W$, the angle of $c_{0}$ at $w$ is $\Theta^{\prime}+X$-large, then the angle of $c$ at $w$ is $\Theta^{\prime}$-large; if, for $w \in W$, the angle of $c_{0}$ at $w$ is $4 X$-small, then the angle of $c$ at $w$ is $5 X$-small.

Since not all $c \in \mathcal{C}$ are $6 X$-small, we conclude from (3.20) that $W \neq \emptyset$. Among all $w \in W$ we let $w_{0}$ be the one closest to $v_{0}$. Then (3.18) implies that $w_{0}$ is also closest to all $h v_{0}, h \in B_{\alpha}(e)$. In particular, any $c$ will, on its way to $\xi$, meet $w_{0}$ before meeting any of the other $w$. Using (3.19) this implies that any geodesic starting in some $h v_{0}, h \in B_{\alpha}(e)$ and ending in $w_{0}$ will be $2 X$-small. If $\Varangle_{w_{0}} c_{0}$ is $4 X$-large, then by (3.20) for any $c \in \mathcal{C}$ the angle of $c$ at $w_{0}$ is $3 X$-large. Using Lemma 3.16 a) we see that in this case, $B_{\alpha}(e) \times\{\xi\} \subseteq V\left(w_{0}, 2 X\right)$ and $\left.\mathrm{b}\right)$ holds.

Therefore we may assume that $\Varangle_{w_{0}} c_{0}$ is $4 X$-small. Then (3.20) implies that for all $c \in \mathcal{C}$ the angle at $w_{0}$ is $5 X$-small. Since not all $c \in \mathcal{C}$ are $\Theta$-small, there are $w \in W$ and $c \in \mathcal{C}$ such that the angle of $c$ at $w$ is $5 X$-large. Among all such pairs we pick $\left(w_{1}, c_{1}\right)$ such that $w_{1}$ is closest to $v_{0}$. As before (3.18) implies that $w_{1}$ is also closest to all $h v_{0}, h \in B_{\alpha}(e)$. Using (3.19) this implies that all geodesics starting in some $h v_{0}, h \in B_{\alpha}(e)$ and ending at $w_{1}$ are $5 X$-small. Since $w_{0} \neq w_{1}$ we can use (3.18) again, to see that for any $h \in B_{\alpha}(e)$ there is a geodesic from $h v_{0}$ to $\xi$ that agrees with $c_{1}$ between $w_{0}$ and $\xi$. Therefore, for any $h \in B_{\alpha}(e)$ there is a geodesic from $h v_{0}$ to $\xi$ for which the angle at $w_{1}$ is $5 X$-large. If $\xi \in V$, then using Lemma 3.15 a), we deduce $B_{\alpha}(e) \times\{\xi\} \subseteq V\left(w_{1}, 5 X\right)$ and b) holds.

If $\xi \in \partial \Gamma$ we need to distinguish two further cases. If the angle of $c_{1}$ at $w_{1}$ is even $\Theta=6 X$-large, then Lemma 3.16 a) implies $B_{\alpha}(e) \times\{\xi\} \subseteq V\left(w_{1}, 5 X\right)$ and b) holds. Otherwise, we use again that not all $c \in \mathcal{C}$ are $6 X$-small. Therefore, there is $w_{2} \in W$ and $c_{2} \in \mathcal{C}$ such that the angle of $c_{2}$ at $w_{2}$ is $6 X$-large. Since $w_{1}$ was chosen closest to $v_{0}, w_{2}$ will be further from $v_{0}$ than $w_{1}$. Now using (3.20) twice we see that for any geodesic $c \in \mathcal{C}$ the angle at $w_{2}$ is $4 X$-large. Since we already found geodesics for any $h \in B_{\alpha}(e)$ from $h v_{0}$ to $\xi$ whose angle at $w_{1}$ is $5 X$-large, we can now use Lemma 3.16 b) to conclude $B_{\alpha}(e) \times\{\xi\} \subseteq V\left(w_{1}, 5 X\right)$. Therefore b) holds.

Covering $G \times \overline{P_{d, \Theta}}$. Associated to $\Gamma$ there is, for given $d>0$ and a given size for angles $\Theta$, a finite dimensional simplicial complex $P_{d, \Theta}$, the relative Rips complex of $\Gamma$. The construction of $P_{d, \Theta}$ is reviewed in Definition $\overline{A .3}$. The union $\overline{P_{d, \Theta}}:=$ $P_{d, \Theta} \cup \partial \Gamma$ carries a compact topology, see Lemma A.11 b) Theorem 3.1 has the following straight forward extension from $\Delta$ to $\overline{P_{d, \Theta}}$. 
Corollary 3.21. For any $d>0$ and any size for angles $\Theta$ the action of $G$ on $\overline{P_{d, \Theta}}$ is finitely $\mathcal{P}$-amenable.

Proof. Given $\alpha>0$ we need to construct a $\mathcal{P}$-cover of $G \times \overline{P_{d, \Theta}}$ as in Definition 0.1

The action of $G$ on $P_{d, \Theta}$ is simplicial and the dimension of $P_{d, \Theta}$ is finite by Lemma A.4 In particular, Remark 0.3 applies and we find a $\mathcal{P}$-cover $\mathcal{W}$ of $G \times P_{d, \Theta}$ of dimension $N^{\prime}$ such that

(3.22) $\operatorname{dim} \mathcal{W}$ is bounded by the dimension of $P_{d, \Theta}$;

(3.23) for any $(g, x) \in G \times P_{d, \Theta}$ there is $W \in \mathcal{W}$ such that $G \times\{x\} \subseteq W$.

The compact topology on $\overline{P_{d, \theta}}$ is such that $P_{d, \Theta}$ is not necessarily open in $\overline{P_{d, \theta}}$, but $P_{d, \Theta} \backslash V_{\infty}$ is open in $\overline{P_{d, \theta}}$. Therefore, the collection $\left\{W \backslash G \times V_{\infty} \mid W \in \mathcal{W}\right\}$ consists of open $\mathcal{P}$-subsets, satisfies (3.22) and satisfies (3.23) for all $(g, x) \in G \times\left(P_{d, \Theta} \backslash V_{\infty}\right)$.

Given $\alpha>0$, Theorem 3.1 provides a $\mathcal{P}$-cover $\mathcal{V}$ for $G \times \Delta$ such that

(3.24) $\operatorname{dim} \mathcal{V}$ is bounded by a number independent of $\alpha$;

(3.25) for any $(g, \xi) \in G \times \Delta$ there is $V \in \mathcal{V}$ such that $B_{\alpha}(g)\{\xi\} \subseteq V$.

Since $\overline{P_{d, \Theta}}$ is compact with a countable basis for the topology it is also metrizable. Therefore we find a $G$-invariant metric $d_{G \times \overline{P_{d, \Theta}}}$ on $G \times \overline{P_{d, \Theta}}$. Using Lemma B.2 we can extend $\mathcal{V}$ to $G$-invariant collection $\mathcal{V}^{+}$of $\mathcal{P}$-subset of $G \times \overline{P_{d, \Theta}}$ that still satisfies (3.24) and (3.25). Altogether $\left\{W \backslash G \times V_{\infty} \mid W \in \mathcal{W}\right\} \cup \mathcal{V}^{+}$is the desired cover of $\overline{G \times \overline{P_{d, \Theta}}}$.

\section{The Farrell-Jones Conjecture for Relatively hyperbolic groups}

Let $G$ be a group and $\mathcal{A}$ be an additive category with a strict $G$-action and a strict direct sum. In [3, Sec. 4.1] such categories are called additive $G$-categories. Similar to (twisted) group rings there is an additive category $\int_{G} \mathcal{A}$ whose morphisms are formal linear combinations of group elements with morphisms from $\mathcal{A}$ as coefficient: $\mathbb{A}$. Given a family $\mathcal{F}$ of subgroups of $G$ there is the assembly map

$$
H_{*}^{G}\left(E_{\mathcal{F}} G ; \mathbf{K}_{\mathcal{A}}\right) \rightarrow K_{*}\left(\int_{G} \mathcal{A}\right) .
$$

The $K$-theoretic Farrell-Jones Conjecture (with coefficients) asserts that this map is an isomorphism for the family $\mathcal{F}:=$ VCyc of virtually cyclic subgroups of $G[9$, Conj. 3.2]. The original formulation of the Conjecture in [20] can be recovered as a special case of (4.1) by using for $\mathcal{A}$ the category of finitely generated free $\mathbb{Z}$-modules; then $\int_{G} \mathcal{A}$ is equivalent to the category of finitely generated free $\mathbb{Z}[G]$-modules. We will say that $G$ satisfies the $K$-theoretic Farrell-Jones Conjecture relative to $\mathcal{F}$ if (4.1) is an isomorphism for all additive $G$-categories $\mathcal{A}$. A consequence of the transitivity principle (see [20, Thm. A.10] and [2, Thm. 2.10]) is the following: Suppose that $G$ satisfies the $K$-theoretic Farrell-Jones Conjecture relative to $\mathcal{F}$ and that every group $F \in \mathcal{F}$ satisfies the $K$-theoretic Farrell-Jones Conjecture. Then $G$ satisfies the $K$-theoretic Farrell-Jones Conjecture.

If $\mathcal{A}$ is in addition equipped with a strict involution, see for example [3. Sec. 4.1], then $\int_{G} \mathcal{A}$ inherits an involution and there is the $L$-theoretic assembly map

$$
H_{*}^{G}\left(E_{\mathcal{F}} G ; \mathbf{L}_{\mathcal{A}}^{-\infty}\right) \rightarrow L_{*}^{\langle-\infty\rangle}\left(\int_{G} \mathcal{A}\right)
$$

The $L$-theoretic Farrell-Jones Conjecture (with coefficients) asserts that this map is an isomorphism for the family of virtually cyclic subgroups VCyc. Everything said for $K$-theory above has an $L$-theory counterpart. In particular, we will say that $G$ satisfies the $L$-theoretic Farrell-Jones Conjecture relative to $\mathcal{F}$ if (4.2) is an isomorphism for all additive $G$-categories $\mathcal{A}$ with involution.

\footnotetext{
${ }^{4}$ In 9 Sec. 2] this category is denoted $\mathcal{A} *_{G} G / G$.
} 
Next we give a minor reformulation of conditions from [3, 6] that implies the Farrell-Jones Conjecture relative to $\mathcal{F}$. For a family $\mathcal{F}$ of groups we write $\mathcal{F}_{2}$ for the family of groups that contain a group from $\mathcal{F}$ as a subgroup of index $\leq 2$.

Theorem 4.3. Let $G$ be a group that admits a finitely $\mathcal{F}$-amenable action on a compact contractible finite-dimensional $A N R \bar{X}$. Then

a) $G$ satisfies the $K$-theoretic Farrell-Jones Conjecture relative to $\mathcal{F}$;

b) $G$ satisfies the L-theoretic Farrell-Jones Conjecture relative to $\mathcal{F}_{2}$.

Proof. We start by recalling [3, Def. 1.5] where a metric space $Y$ is called controlled $N$-dominated if for every $\varepsilon>0$ there is a finite $C W$-complex $K$ of dimension at most $N$, maps $i: Y \rightarrow K, p: K \rightarrow Y$ and a homotopy $H: Y \times[0,1] \rightarrow Y$ between $p \circ i$ and $\operatorname{id}_{Y}$ such that the tracks $\{H(y, t) \mid t \in[0,1]\}$ are of diameter at most $\varepsilon$. Compact finite dimensional $A N R$ s have this property; this follows for example from [12, Thm. 10.1, p.122].

a) In [6, Thm. 1.1] the $K$-theoretic Farrell-Jones Conjecture relative to $\mathcal{F}$ is proven under very similar conditions. In this reference also an action of $G$ on a compact metrizable space $\bar{X}$ is required. Assumption 1.4 in this reference is what we defined as finitely $\mathcal{F}$-amenability here. In this reference it is further assumed that $\bar{X}$ contains a simplicial complex $X$ whose complement is a $Z$-set in $\bar{X}$. This further assumption is only used in the proof of [6, Lem. 6.9]. It is not hard to see that this Lemma also holds for controlled $N$-dominated metric spaces, compare [3, Lem. 8.4]. Therefore, the $Z$-set assumption can be safely replaced with the assumption that $\bar{X}$ is a compact finite-dimensional $A N R$. Alternatively, a) can be deduced from [41, Thm 1.1]. The conditions given in this reference are more involved (using strong homotopy actions) and designed for more general situations, but can be checked to hold in our case.

b) This follows from [3, Thm. 1.1(ii)]. The assumption in this reference is that $G$ is transfer reducible over $\mathcal{F}$ [3, Def. 1.8]. To check that this assumption is satisfied in our situation we use the action of $G$ on $\bar{X}$. The covers appearing in [3, Def. 1.8] exist because this action is finitely $\mathcal{F}$-amenable.

Theorem 4.4. Let $\mathcal{P}$ be a family of subgroups of $G$ that contains all virtually cyclic subgroups. Suppose that $G$ is relatively hyperbolic to subgroups $P_{1}, \ldots, P_{n} \in \mathcal{P}$. Then

a) $G$ satisfies the $K$-theoretic Farrell-Jones Conjecture relative to $\mathcal{P}$;

b) $G$ satisfies the L-theoretic Farrell-Jones Conjecture relative to $\mathcal{P}_{2}$.

Proof. Let $\overline{P_{d, \Theta}}$ be the space from Definition A.9. By Corollary 3.21 this space carries a finitely $\mathcal{P}$-amenable action. It is a finite-dimensional compact contractible $A N R$ for suitable $d, \Theta$ by Lemma A.11 and Theorem A.16. Now use Theorem 4.3 .

Remark 4.5. In Theorem4.4 it suffices to assume that $\mathcal{P}$ contains all virtually cyclic subgroups of type $I$, instead of all virtually cyclic subgroup. The reason for this is that no virtually cyclic subgroups of type $I I$ appeared in the previous Sections, see Remark 2.18.

For $L$-theory this does not strengthen Theorem 4.4, since all virtually cyclic groups contain a virtually cyclic group of type $I$ as a subgroup of index at most 2 .

For $K$-theory this slightly strengthens Theorem 4.4. However, it is known that such a strengthening is always possible: in the $K$-theoretic Farrell-Jones Conjecture only virtually cyclic subgroups of type $I$ are needed [16].

Corollary 4.6. Let $G$ be relatively hyperbolic to subgroups $P_{1}, \ldots, P_{n}$. Then 
a) $G$ satisfies the $K$-theoretic Farrell-Jones Conjecture, provided that the $P_{i}$ satisfies the $K$-theoretic Farrell-Jones Conjecture;

b) $G$ satisfies the L-theoretic Farrell-Jones Conjecture, provided that all subgroups of $G$ that contain one of the $P_{i}$ as a subgroup of index at most 2 satisfy the L-theoretic Farrell-Jones Conjecture.

Proof. This follows from Theorem 4.4 and the transitivity principle reviewed earlier in this section.

Remark 4.7. Many classes of groups that are known to satisfy the Farrell-Jones Conjecture are closed under finite index overgroups, but there is no general result to this effect. A good formalism to circumvent this difficulty is the Farrell-Jones Conjecture with wreath products considered for example in 8, 21.

A group $G$ is said to satisfy the Farrell-Jones Conjecture with wreath products relative to a family of subgroups $\mathcal{F}$ if for any finite group $F$ the wreath product $G$ ? $F$ satisfies the Farrell-Jones Conjecture relative to $\mathcal{F}$. This version of the conjecture passes to finite overgroups and to finite wreath products [8, Rem. 6.2]. Moreover, the conditions that we verified for relatively hyperbolic groups in the proof of Theorem 4.4 can also be used to obtain results for the Farrell-Jones Conjecture with wreath products [8, Thm. 5.1]. Combining this observation with the transitivity principle we obtain the following variant of Corollary 4.6. Suppose that $G$ is realtively hyperbolic to subgroups $P_{1}, \ldots, P_{n}$ all of which satisfy the Farrell-Jones Conjecture with wreath products, then $G$ satisfies the Farrell-Jones Conjecture with wreath products.

Here it is no longer necessary to distinguish between $K$-and $L$-Theory; everything in this remark applies as stated to the $K$-theoretic and the $L$-theoretic version of the conjecture.

Remark 4.8. If $G$ is relatively hyperbolic to infinite subgroups $P_{1}, \ldots, P_{n}$, then for the action of $G$ on $\Delta$ each $P_{i}$ fixes a unique point in $\Delta$. In particular, no overgroups of the $P_{i}$ have fixed points on the space $P_{2}(\Delta)$ of unordered pairs in $\Delta$ used in 3 , Sec. 9]. It seems plausible that this observation together with a careful analysis of the arguments in [3] can be used to show that the appearance of index 2 overgroups in Theorem 4.4 b) and Corollary 4.6 b) is not necessary.

We conclude this section with some examples where Corollary 4.6 applies.

Example 4.9. Let $G$ be the fundamental group of a complete Riemannian manifold $M$ of pinched negative curvature and finite volume. Then $G$ is hyperbolic relative to virtually finitely generated nilpotent groups [13, 18, Since virtually finitely generated nilpotent groups satisfy both the $K$ - and $L$-theoretic Farrell-Jones Conjecture [2], the $K$ - and $L$-theoretic Farrell-Jones Conjecture for $G$ holds.

Example 4.10. Let $G$ be the fundamental group of a finite graph of groups with finite edge groups. Then the associated action of $G$ on the Bass-Serre tree reveals $G$ as relatively hyperbolic to the vertex groups. Thus if all vertex groups satisfy the $K$-theoretic Farrell-Jones Conjecture then $G$ satisfies the $K$-theoretic FarrellJones Conjecture. If all overgroups of vertex groups of index at most 2 satisfy the $L$-theoretic Farrell-Jones Conjecture then $G$ satisfies the $L$-theoretic Farrell-Jones Conjecture.

Example 4.11. Suppose that $G$ acts cocompactly and properly discontinuously on a systolic complex with the Isolated Flats Property. Then $G$ is relatively hyperbolic to virtually finitely generated abelian subgroups [17. Since virtually finitely generated abelian groups satisfy both the $K$ - and $L$-theoretic Farrell-Jones Conjecture [2, 37, the $K$ - and $L$-theoretic Farrell-Jones Conjecture for $G$ holds. 


\section{Appendix A. The Relative Rips COMplex And the BOUndary}

In this appendix we prove that $\Delta$ is finite dimensional and embeds into a finite dimensional compact contractible $A N R$ with a complement homeomorphic to a simplicial complex 5 . For the boundary of hyperbolic groups both these facts are well-known [11]. For relative hyperbolic groups closely related results have been obtained by Dahmani [15] and Mineyev-Yaman [30. Our treatment is very similar to the one in these references, but we do not require any assumptions on the parabolic subgroups.

Throughout this appendix we use again the notation from Section 2 In particular, $G$ is a relatively hyperbolic group exhibited by a cocompact action on the fine and hyperbolic graph $\Gamma$ with finite edge stabilizers. Throughout this appendix we will make the following additional assumption on $\Gamma$ : no two vertices from $V_{\infty}$ are adjacent. This can be easily arranged for by replacing $\Gamma$ with its first barycentric subdivision.

\section{$\Delta_{+}$is finite dimensional.}

Theorem A.1. The dimension of $\Delta_{+}$is finite.

A very similar result by Dahmani is [15, Lemma 3.7], whose proof we mostly copy.

Proof. For a vertex $v$ of finite valency let $\partial_{v} \Gamma \subseteq \partial \Gamma$ consist of all $\xi \in \partial \Gamma$ for which there exists a $\Theta^{(3)}$-small geodesic from $v$ to $\xi$. Let $U$ be the union of all $\partial_{v} \Gamma$, where we vary $v$ over all vertices of finite valency. If $\xi \in \partial \Gamma \backslash U$, then any geodesic from a vertex of finite valency to $\xi$ will have a $\Theta^{(3)}$-large angle at infinitely many vertices. The finite union of finite dimensional spaces is again finite dimensional, see 25. p.28]. Therefore it suffices to show that $V, U$ and $\partial \Gamma \backslash U$ are finite dimensional subspace of $\Delta_{+}$.

We recall again the countable sum theorem [36. Thm. 2.5, p.125]: the countable union of closed subsets of dimension $\leq n$ is of dimension $\leq n$. In particular, the countable subspace $V \subseteq \Delta_{+}$is of dimension 0 . The spaces $\partial_{v} \Gamma$ are finite dimensional by Lemma A.2 below with a uniform bound on their dimensions. As a consequence of Addendum 2.10 the $\partial_{v} \Gamma$ are closed in $\partial \Gamma$. Thus $U$ is finite dimensional by the countable sum theorem.

It remains to show that $\partial \Gamma \backslash U$ is finite dimensional. In fact, we will show that it is homeomorphic to a subset of the boundary of a tree $T$ and therefore 0 -dimensional. Fix a vertex $v_{0}$ of finite valency. The tree $T$ is a maximal subtree of $\Gamma$ and can be build inductively by choosing, for each vertex at distance $n$ from $v_{0}$, an edge of $\Gamma$ that connects it to a vertex at distance $n-1$ from $v_{0}$. For any $\xi \in \partial \Gamma$ the tree $T$ will contain a geodesic from $v_{0}$ to $\xi$. (To construct such a geodesic pick a sequence of vertices $v_{n}$ with $v_{n} \rightarrow \xi$ and apply Lemma 2.9 a) to geodesics $c_{n}$ from $v_{0}$ to $v_{n}$ in $T$.) The inclusion $T \rightarrow \Gamma$ induces a continuous surjective map $f: \Delta_{+}(T) \rightarrow \Delta_{+}$, where $\Delta_{+}(T)$ is the union of the vertices of $T$ with $\partial T$ and is also equipped with the observer topology. For any $\xi \in \partial \Gamma \backslash U$ there is a unique geodesic in $T$ from $v_{0}$ to $\xi$. Indeed, any geodesic $c$ in $\Gamma$ from $v_{0}$ to $\xi$ will have a $\Theta^{(3)}$-large angle at infinitely many vertices and any other geodesic $c^{\prime}$ in $\Gamma$ from $v_{0}$ to $\xi$ will, by Lemma 2.11 also pass through these vertices. Therefore $c=c^{\prime}$ if both are in $T$. It follows that the restriction of $f$ to the preimage of $\partial \Gamma \backslash U$ is bijective. We claim that, since $\Delta_{+}(T)$ is compact, this restriction of $f$ is a homeomorphism. To prove this claim we need to show that the inverse of $f$ on $\partial \Gamma \backslash U$ is continuous. Let $\xi_{n} \rightarrow \xi$ be a convergent sequence in $\partial \Gamma \backslash U$. Let $\xi_{n}^{\prime}, \xi^{\prime}$ be the unique preimages in $\Delta_{+}(T)$ of the $\xi_{n}, \xi$. We

\footnotetext{
${ }^{5}$ The complement will be the relative Rips complex minus its vertices of infinite valence. Thus the complement is not a subcomplex of the relative Rips complex, but it is homeomorphic to a simplicial complex
} 
need to show $\xi_{n}^{\prime} \rightarrow \xi^{\prime}$ in $\Delta_{+}(T)$. Assume this fails. Then, as $\Delta_{+}(T)$ is compact, there is a subsequence $I \subseteq \mathbb{N}$ with $\lim _{n \in I} \xi_{n}^{\prime}=\xi^{\prime \prime} \neq \xi^{\prime}$ in $\Delta_{+}(T)$. By continuity of $f$, we have $f\left(\xi^{\prime \prime}\right)=\lim _{n \in I} f\left(\xi_{n}^{\prime}\right)=\lim _{n \in I} \xi_{n}=\xi$. In particular, $\xi^{\prime \prime}$ belongs to the preimage of $\partial \Gamma \backslash U$ under $f$. Now we use that the restriction of $f$ to this preimage is injective to contradict $\xi^{\prime \prime} \neq \xi^{\prime}$. Thus $\xi_{n}^{\prime} \rightarrow \xi^{\prime}$ in $\Delta_{+}(T)$.

Lemma A.2. There is $N$ such that $\operatorname{dim} \partial_{v_{0}} \Gamma \leq N$ for all vertices $v_{0}$ of finite valency.

Proof. Let $V_{v_{0}}$ be the set of all vertices $v$ of $\Gamma$ for which there exists a $\Theta^{(3)}{ }_{\text {-small }}$ geodesic from $v_{0}$ to $v$. Hyperbolicity of $\Gamma$ implies that this is a quasi-convex subset of $\Gamma$ : there is $r>0$ such that for $v, v^{\prime} \in V_{v_{0}}$ any geodesic between $v$ and $v^{\prime}$ will be contained in the $r$-neighborhood of the union of geodesics from $v_{0}$ to $v$ and $v^{\prime}$. Consequently, $V_{v_{0}}$ is hyperbolic in the metric $d_{v_{0}}$ inherited as a subspace of $\Gamma$. Let $\Gamma_{v_{0}}$ be the graph whose vertices are $V_{v_{0}}$ and for which there is an edge between $v$ and $v^{\prime}$ whenever $d_{v_{0}}\left(v, v^{\prime}\right) \leq 2 r+1$. Of course, the metric $d_{\Gamma_{v_{0}}}$ induced by $\Gamma_{v_{0}}$ on $V_{v_{0}}$ satisfies $d_{v_{0}} \leq(2 r+1) d_{\Gamma_{v_{0}}}$. Conversely, since any $\Theta^{(3)}$-small geodesic in $\Gamma$ starting in $v_{0}$ defines also a path in $\Gamma_{v_{0}}$, we can as before apply hyperbolicity to any geodesic $c$ in $\Gamma$ between points $v, v^{\prime} \in V_{v_{0}}$ and replace $c$ with a path in $\Gamma_{v_{0}}$ of length equal to the length of $c$. Thus $d_{\Gamma_{v_{0}}}$ and $d_{v_{0}}$ are Lipschitz equivalent. In particular, $\Gamma_{v_{0}}$ is hyperbolic.

Let $v, v^{\prime} \in V_{v_{0}}$. Let $\Theta$ be a size for angles such that $3 \Theta^{(3)} \subseteq \Theta$. Since $v_{0}$ is a vertex of finite valency we can, after enlarging $\Theta$, assume that all angles at the vertex $v_{0}$ are $\Theta$-small. We claim that any geodesic $c$ in $\Gamma$ between $v$ and $v^{\prime}$ is $\Theta$-small. If this fails, then for some internal vertex $w$ of $c$ the angle $\Varangle_{w} c$ is $\Theta$-large. Let $c_{v}$ and $c_{v^{\prime}}$ be $\Theta^{(3)}$-small geodesics from $v_{0}$ to $v$ and $v^{\prime}$. We apply Lemma 2.12 to the geodesic triangle whose sides are $c, c_{v}$ and $c_{v^{\prime}}$. Since $c_{v}$ and $c_{v}^{\prime}$ are $\Theta^{(3)}$-small we conclude that $w=v_{0}$. But our choice of $\Theta$ guarantees that $\Varangle_{w} c$ is $\Theta$-small. Therefore $c$ is $\Theta$-small.

For $v \in V_{v_{0}}$ the ball $B_{r}(v)$ of radius $r$ around $v$ in $V_{v_{0}}$ with respect to $d_{v_{0}}$ is contained in the set of vertices of $\Gamma$ that can be connected to $v$ by a $\Theta$-small geodesic of length $r$. If $v$ is of finite valency, then this set is finite by Corollary 2.2, Moreover, as the action of $G$ on the vertices of $\Gamma$ is cofinite, the number of vertices in $B_{r}(v)$ is bounded by a number depending only on $r$. Any vertex $w \in V_{v_{0}}$ of infinite valency is adjacent to a vertex $v \in V_{v_{0}}$ of finite valency. (Consider the $\Theta^{(3)}$ small geodesic between $w$ and $v_{0}$ and recall that we assumed that no two vertices of infinite valency are adjacent.) Since $B_{r}(w) \subseteq B_{r+1}(v)$ whenever $v, w \in V_{v_{0}}$ are adjacent, we can now conclude that $V_{v_{0}}$ is uniformly proper with respect to $d_{v_{0}}$ (or $d_{\Gamma_{v_{0}}}$ ): the number of elements in a ball is bounded by a number only depending on the radius of the ball. The dimension of the boundary of a hyperbolic graph can be estimated in terms of the number of vertices in balls of a fixed radius (depending on the hyperbolicity constant); this is a standard fact see for example [5. Proof of Prop.9.3(ii)]. Consequently, the boundary of $\Gamma_{v_{0}}$ is finite dimensional. Since this boundary agrees with $\partial_{v_{0}} \Gamma$ it follows that $\partial_{v_{0}} \Gamma$ is finite dimensional. The action of $G$ on the set of vertices of finite valency is cocompact. It follows that the maximum of the dimension of the $\partial_{v_{0}} \Gamma$ is finite.

The relative Rips complex. We will say that a geodesic is $(d, \Theta)$-small if it is $\Theta$-small and of length at most $d$.

Definition A.3. Let $\Theta$ be a size for angles and $d>0$. The relative Rips complex $P_{d, \Theta}$ of $\Gamma$ has $V$ as the set of vertices. A finite set $\sigma$ of vertices spans a simplex for $P_{d, \Theta}$ if and only if between any two vertices $v, v^{\prime} \in \sigma$ there exists a $(d, \Theta)$-small geodesic. 
Lemma A.4. The relative Rips complex $P_{d, \Theta}$ is finite dimensional. For $n \geq k \geq 1$ each $k$-simplex is contained in only finitely many $n$-simplices.

Proof. Let $v \in V$ and $e$ be an edge incident to $v$. Because of Lemma 2.1 there are only finitely many vertices $v^{\prime}$ for which there exists a $(d, \Theta)$-small geodesic from $v$ to $v^{\prime}$ whose first edge is $e$. Since $G$ acts cocompactly on the set of edges the number of such geodesics is uniformly bounded.

Fix $v^{\prime} \neq v$ such that there exists a $\Theta$-small geodesic $c$ between $v$ and $v^{\prime}$. Let $W\left(v, v^{\prime}\right)$ be the set of vertices $w$ for which there exist $(d, \Theta)$-small geodesics, $c_{w}$ between $w$ and $v$ and $c_{w}^{\prime}$ between $w$ and $v^{\prime}$. We need to show that the number of elements in $W\left(v, v^{\prime}\right)$ is uniformly bounded. Let $e$ be the initial edge of $c$, starting at $v$. Let $f$ be the initial edge of $c_{w}$, starting at $v$. We claim that $(e, f)$ is $\Theta+2 \Theta^{(3)}$ small. Lemma 2.1 implies then that the number of such $f$ is uniformly bounded and the first paragraph of this proof implies then that the cardinality of $W\left(v, v^{\prime}\right)$ is uniformly bounded.

It remains to prove that $(e, f)$ is $\Theta+2 \Theta^{(3)}$ small. If $c_{w}^{\prime}$ does not meet $v$, then $(e, f)$ is $\Theta^{(3)}$ small. Otherwise $\Varangle_{v} c_{w}^{\prime}$ is $\Theta$-small and Lemma 2.6 implies then that $(e, f)$ is $\Theta+2 \Theta^{(3)}$-small.

In the next statement the positive integer $\delta$ will be again a hyperbolicity constant for $\Gamma$.

Proposition A.5. Assume that $d \geq 4 \delta$ and $7 \Theta^{(3)} \subseteq \Theta$. Then $P_{d, \Theta}$ is contractible. More precisely the following holds:

Let $K$ be a finite subcomplex of $P_{d, \Theta}$. Let $L$ be the subcomplex of $P_{d, \Theta}$ spanned by all vertices $\tilde{v}$ for which there are vertices $v, v^{\prime} \in K$ such that $\tilde{v}$ belongs to a geodesic between $v$ and $v^{\prime}$. Then $K$ is contractible within $L$.

Proof. Let $V_{K}$ be the set of vertices of $K$. Fix $v_{0} \in V_{K}$. Let $\alpha:=\max _{v \in V_{K}} d_{\Gamma}\left(v_{0}, v\right)$. Let $a$ be the number of vertices in $V_{K}$ for which $d_{\Gamma}\left(v_{0}, v\right)=\alpha$. For $v \in V_{K}$ let $W(v)$ be the set of vertices $w \in V$ such that there exists a geodesic $c$ from $v_{0}$ to $v$ such that $c$ passes through $w$ such that $\Varangle_{w} c$ is $2 \Theta^{(3)}$-large. Let $\beta:=$ $\max _{v \in V_{K}, w \in W(v)} d_{\Gamma}\left(v_{0}, w\right)$. Let $b$ be the number of vertices $v \in V_{K}$ for which there is $w \in W(v)$ with $d_{\Gamma}\left(v_{0}, w\right)=\beta$. Clearly, $\alpha \geq \beta$. If $\alpha=0$, then $K$ consists of a single vertex and there is nothing to prove.

For the general case we will use induction on $(\alpha+\beta, a+b)$. We claim that there is a vertex $v \in V_{K}$ and a vertex $\tilde{v}$ on a geodesic from $v_{0}$ to $v$ such that:

(A.6) there is a $(d, \Theta)$-small geodesic from $v$ to $\tilde{v}$;

(A.7) if there is a $(d, \Theta)$-small geodesic from $v$ to $v^{\prime}$, with $v^{\prime} \in V_{K}$, then there is also a $(d, \Theta)$-small geodesic from $\tilde{v}$ to $v^{\prime}$

(A.8) either $d_{\Gamma}\left(v_{0}, v\right)=\alpha$ and $d_{\Gamma}\left(v_{0}, \tilde{v}\right)<\alpha$ or there is $w \in W(v)$ with $d_{\Gamma}\left(v_{0}, w\right)=$ $\beta$, and $d_{\Gamma}\left(v_{0}, \tilde{w}\right)<\beta$ for all $\tilde{w} \in W(\tilde{v})$. (In fact, in the second case we will use $\tilde{v}=w$.

Given this claim there is a homotopy in $L$ that replaces $v$ by $\tilde{v}$. The effect on $(\alpha+\beta, a+b)$ is then that we either reduce $\alpha+\beta$ or that we reduce $a+b$ but do not increase $\alpha+\beta$. This will complete the induction step modulo our claim.

To prove the claim, we distinguish two cases. In the first case we assume $\alpha \geq$ $\beta+d$. Then choose $v \in V_{K}$ such that $d_{\Gamma}\left(v_{0}, v\right)=\alpha$. As $\delta$ is an integer, we can pick a vertex $\tilde{v}$ with $d_{\Gamma}(v, \tilde{v})=2 \delta$ that belongs to a geodesic $c$ between $v_{0}$ and $v$. Clearly (A.8) holds. The geodesic $\left.c\right|_{[v, \tilde{v}]}$ is of length $2 \delta \leq d$. Let $w$ be an internal vertex of $\left.c\right|_{[v, \tilde{v}]}$. If $\Varangle_{w} c$ is $\Theta$-large, then, since $2 \Theta^{(3)} \subseteq \Theta, w \in W(v)$ and $\beta \geq d_{\Gamma}\left(v_{0}, w\right)>d_{\Gamma}\left(v_{0}, \tilde{v}\right) \geq \alpha-2 \delta \geq \alpha-d$. Since this contradicts $\alpha \geq \beta+d$, the angle $\Varangle_{w} c$ at $w$ is $\Theta$-small. Thus $\left.c\right|_{[v, \tilde{v}]}$ is $\Theta$-small and (A.6) holds. In fact, this argument proves that $\left.c\right|_{[v, \tilde{v}]}$ is $2 \Theta^{(3)}$-small. To prove (A.7) consider $v^{\prime} \in V_{K}$ 
such that there exists a $(d, \Theta)$-small geodesic $c^{\prime}$ between $v$ and $v^{\prime}$. Hyperbolicity of $\Gamma$ implies that there is $w \in V$ with $d_{\Gamma}(\tilde{v}, w) \leq \delta$ such that $w$ belongs to a geodesic between $v_{0}$ and $v^{\prime}$ or to a geodesic between $v$ and $v^{\prime}$. In the first case $d_{\Gamma}\left(w, v^{\prime}\right)=d_{\Gamma}\left(v_{0}, v^{\prime}\right)-d_{\Gamma}\left(w, v_{0}\right) \leq d_{\Gamma}\left(v_{0}, v^{\prime}\right)-\left(d_{\Gamma}\left(v_{0}, \tilde{v}\right)-d_{\Gamma}(w, \tilde{v})\right) \leq \alpha-(\alpha-$ $2 \delta-\delta)=3 \delta \leq d-\delta$. In the second case $d_{\Gamma}\left(w, v^{\prime}\right)=d_{\Gamma}\left(v, v^{\prime}\right)-d_{\Gamma}(w, v) \leq$ $d_{\Gamma}\left(v, v^{\prime}\right)-\left(d_{\Gamma}(v, \tilde{v})-d_{\Gamma}(w, \tilde{v})\right) \leq d-(2 \delta-\delta)=d-\delta$. Thus $d_{\Gamma}\left(\tilde{v}, v^{\prime}\right) \leq d$ in both cases. Let $\tilde{c}$ be a geodesic from $v^{\prime}$ to $\tilde{v}$. It remains to show that $\tilde{c}$ is $\Theta$-small. Assume this fails. Then there is an internal vertex $w$ of $\tilde{c}$ such that $\Varangle_{w} \tilde{c}$ is $\Theta$-large. We can then apply Lemma 2.12 to the geodesics $\left.c\right|_{[v, \tilde{v}]}, c^{\prime}$ and $\tilde{c}$. Since $\left.c\right|_{[v, \tilde{v}]}$ is $2 \Theta^{(3)}$-small and $2 \Theta^{(3)}+2 \Theta^{(3)} \subseteq \Theta$, Lemma 2.12 implies $w \in c^{\prime}$. In particular, $d_{\Gamma}(w, v)<d$ and therefore $d_{\Gamma}\left(v_{0}, w\right) \geq d_{\Gamma}\left(v_{0}, v\right)-d_{\Gamma}(w, v)>\alpha-d \geq \beta$. Let $c^{\prime \prime}$ be a geodesic from $v^{\prime}$ to $v_{0}$. Consider the geodesic triangle whose sides are $\tilde{c}, c^{\prime \prime}$ and $\left.c\right|_{\left[v_{0}, \tilde{v}\right]}$. Since $\Varangle_{w} \tilde{c}$ is $\Theta$-large, we can again apply Lemma 2.12 and deduce $\left.w \in c^{\prime \prime} \cup c\right|_{\left[v_{0}, \tilde{v}\right]}$. Moreover, since $2 \cdot 2 \Theta^{(3)}+3 \Theta^{(3)} \subseteq \Theta$ we can combining this Lemma with Lemma 2.13 and deduce that the angle of $c^{\prime \prime}$ or of $\left.c\right|_{\left[v_{0}, \tilde{v}\right]}$ at $w$ will be $2 \Theta^{(3)}$-large. In the first case $w \in W\left(v^{\prime}\right)$ in the second $w \in W(v)$. Both cases imply $d_{\Gamma}\left(w, v_{0}\right) \leq \beta$. But this is a contradiction since we proved earlier $d_{\Gamma}\left(v_{0}, w\right)>\beta$.

It remains to prove our claim under the assumption $\alpha<\beta+d$. If $\beta=0$ then there are $(d, \Theta)$-small geodesics between $v_{0}$ and any $v \in V_{K}$. Thus we can set $\tilde{v}:=v_{0}$ if $\beta=0$. So assume $\beta>0$ and pick $v \in V_{K}$, a geodesic $c$ between $v_{0}$ and $v$ and an internal vertex $\tilde{v}$ of $c$ such that $\Varangle_{\tilde{v}} c$ is $2 \Theta^{(3)}$-large and $d_{\Gamma}\left(v_{0}, \tilde{v}\right)=\beta$. Clearly (A.8) holds. The geodesic $\left.c\right|_{[v, \tilde{v}]}$ is of length $\leq \alpha-\beta \leq d$. Let $w$ be an internal vertex of $\left.c\right|_{[v, \tilde{v}]}$. If $\Varangle_{w} c$ is $\Theta$-large, then, since $2 \Theta^{(3)} \subseteq \Theta, w \in W(v)$ and we obtain the contradiction $\beta \geq d_{\Gamma}\left(v_{0}, w\right)>d_{\Gamma}\left(v_{0}, \tilde{v}\right)=\beta$. Thus $\left.c\right|_{[v, \tilde{v}]}$ is $\Theta$-small and A.6 holds. In fact, this argument proves that $\left.c\right|_{[v, \tilde{v}]}$ is $2 \Theta^{(3)}$-small. To prove (A.7) let $v^{\prime} \in V_{K}$ such that there exists a $(d, \Theta)$-small geodesic $c^{\prime}$ between $v$ and $v^{\prime}$. Let $c^{\prime \prime}$ be a geodesic between $v_{0}$ and $v^{\prime}$. Consider the geodesic triangle whose sides are $c$, $c^{\prime}$ and $c^{\prime \prime}$. Since $\Varangle_{\tilde{v}} c$ is $2 \Theta^{(3)}$-large, Lemma 2.12 implies $\tilde{v} \in c^{\prime} \cup c^{\prime \prime}$. If $\tilde{v} \in c^{\prime}$, then $\left.c^{\prime}\right|_{\left[v^{\prime}, \tilde{v}\right]}$ is a $(d, \Theta)$-small geodesic between $v^{\prime}$ and $\tilde{v}$ and (A.7) holds. If $\tilde{v} \in c^{\prime \prime}$, then we use the restriction $\left.c^{\prime \prime}\right|_{\left[v^{\prime}, \tilde{v}\right]}$. This restriction is $\Theta$-small, since otherwise we could argue as in the proof of (A.6) and find $w \in W\left(v^{\prime}\right)$ with $d_{\Gamma}\left(v_{0}, w\right)>\beta$. Similarly, in this case $d_{\Gamma}\left(\tilde{v}, v^{\prime}\right)=d_{\Gamma}\left(v^{\prime}, v_{0}\right)-d_{\Gamma}\left(\tilde{v}, v_{0}\right) \leq \alpha-\beta \leq d$.

From now on we will assume that $d$ and $\Theta$ satisfy the assumptions of Proposition A.5.

Definition A.9. Let $\overline{P_{d, \Theta}}:=P_{d, \Theta} \cup \partial \Gamma$. For an open subset $U$ of $\Delta_{+}$we define $P_{d, \Theta}(U) \subseteq P_{d, \Theta}$ as the subcomplex spanned by the vertices of $\Gamma$ that belong to $U$. For $\xi \in \overline{P_{d, \Theta}}$ we define collections of subsets $\mathcal{N}_{\xi}$ of $\overline{P_{d, \Theta}}$ as follows.

a) If $\xi \in P_{d, \Theta} \backslash V_{\infty}$, then $\mathcal{N}_{\xi}$ consists of all open neighborhoods $W$ of $\xi$ in $P_{d, \Theta} \backslash V_{\infty}$

b) If $\xi \in \partial \Gamma$, then $\mathcal{N}_{\xi}$ consists of all sets of the form $P_{d, \Theta}(U) \cup U$, where $U$ is a neighborhood of $\xi$ in $\Delta_{+}$.

c) If $\xi \in V_{\infty}$, then $\mathcal{N}_{\xi}$ consists of all sets of the form $P_{d, \Theta}(U) \cup U \cup W$, where $U$ is a neighborhood of $\xi$ in $\Delta_{+}$, and $W$ is a neighborhood of $\xi$ in $P_{d, \Theta}$ (i.e., the intersection of $W$ with any simplex of $P_{d, \Theta}$ is open in the simplex).

We will use the topology on $\overline{P_{d, \Theta}}$ which for $U \subseteq \overline{P_{d, \Theta}}$ is open if and only if for every $\xi \in U$ there is $N \in \mathcal{N}_{\xi}$ with $N \subseteq U$.

Lemma A.10. Each $N \in \mathcal{N}_{\xi}$ is a (not necessarily open) neighborhood of $\xi$ in $\overline{P_{d, \Theta}}$.

Proof. If $\xi \in P_{d, \Theta} \backslash V_{\infty}$, then $\mathcal{N}_{\xi}$ consists of open subsets of $\overline{P_{d, \Theta}}$. Before we discuss the cases $\xi \in \partial \Gamma$ and $\xi \in V_{\infty}$ we point out a collection of open subsets of $\overline{P_{d, \Theta}}$. 
Consider $U^{\prime} \subseteq \Delta_{+}$open, $W^{\prime} \subseteq P_{d, \Theta}$ open and assume that $P_{d, \Theta}\left(U^{\prime}\right) \subseteq W^{\prime}$ and that $V_{\infty} \cap W^{\prime} \subseteq U^{\prime}$. It is not difficult to check that then $W^{\prime} \cup U^{\prime}$ is open in $\overline{P_{d, \Theta}}$.

Let $\xi \in \partial \Gamma$ and $N=P_{d, \Theta}(U) \cup U \in \mathcal{N}_{\xi}$. Lemma 2.19 implies that there exists a neighborhood $U^{\prime}$ of $\xi$ in $\Delta_{+}$with the following property: if $v$ is a vertex in $\Gamma$ for which there exists a geodesic of length $\leq d$ starting in $v$ and ending in $U^{\prime}$, then $v \in U$. It follows that $P_{d, \Theta}\left(U^{\prime}\right)$ is contained in the interior $P_{d, \Theta}^{\circ}(U)$ of $P_{d, \Theta}(U)$. Set now $W^{\prime}:=P_{d, \Theta}^{\circ}(U) \backslash\left(V_{\infty} \backslash U^{\prime}\right)$, i.e., we remove all vertices of infinite valency from $P_{d, \Theta}(U)$ that do not belong to $U^{\prime}$. Then $W^{\prime} \cup U^{\prime}$ satisfies the conditions from the first paragraph of the proof and thus is open in $\overline{P_{d, \Theta}}$. Since $\xi \in W^{\prime} \cup U^{\prime} \subseteq N, N$ is a neighborhood of $\xi$.

Let $\xi \in V_{\infty}$ and $N=P_{d, \Theta}(U) \cup U \cup W \in \mathcal{N}_{\xi}$. Addendum 2.20 implies that there exists a neighborhood $U^{\prime}$ of $\xi$ in $\Delta_{+}$with the following property: if $v$ is a vertex in $\Gamma$ for which there exists a $(d, \Theta)$-small geodesic starting in $v$ and ending in $U^{\prime} \backslash \xi$, then $v \in U$. It follows that $P_{d, \Theta}\left(U^{\prime}\right) \backslash\{\xi\}$ is contained in $P_{d, \Theta}^{\circ}(U)$. Thus $P_{d, \Theta}\left(U^{\prime}\right) \subseteq P_{d, \Theta}^{\circ}(U) \cup W$. Set $W^{\prime}:=\left(P_{d, \Theta}^{\circ}(U) \cup W\right) \backslash\left(V_{\infty} \backslash U^{\prime}\right)$. Again $W^{\prime} \cup U^{\prime}$ is open in $\overline{P_{d, \Theta}}$. Since $\xi \in W^{\prime} \cup U^{\prime} \subseteq N, N$ is a neighborhood of $\xi$.

Lemma A.10 implies that the inclusions $\Delta \rightarrow \overline{P_{d, \Theta}}$ and $P_{d, \Theta} \backslash V_{\infty} \rightarrow \overline{P_{d, \Theta}}$ are homeomorphisms onto their images. The canonical map $i: P_{d, \Theta} \rightarrow \overline{P_{d, \Theta}}$ is continuous, but $i$ is not a homeomorphism onto its image (unless $V_{\infty}=\emptyset$ ). For the continuity of $i$ it is important to include the sets $W$ in the definition of the neighborhood basis $\mathcal{N}_{\xi}$ for $\xi \in V_{\infty}$, see Definition A.9 c).

Lemma A.11. a) The topology on $\overline{P_{d, \Theta}}$ is second countable;

b) $\overline{P_{d, \Theta}}$ is compact and metrizable;

c) $\overline{P_{d, \Theta}}$ is finite dimensional;

d) for any $\xi \in \overline{P_{d, \Theta}}$, and every neighborhood $U$ of $\xi$ in $\overline{P_{d, \Theta}}$ there is a neighborhood $U^{\prime}$ of $\xi$ in $\overline{P_{d, \Theta}}$ such that for any map $f: S^{k} \rightarrow i^{-1}\left(U^{\prime}\right)$ there is a map $\hat{f}: D^{k+1} \rightarrow i^{-1}(U)$ such that $\left.\hat{f}\right|_{S^{k}}=f$.

Proof. a) Since $G$ is countable and acts cocompactly on $\Gamma$ there are only countably many simplices in $P_{d, \Theta}$. By Lemma A.4 every point in $P_{d, \Theta} \backslash V_{\infty}$ is contained in only finitely many simplices. Therefore, $P_{d, \Theta} \backslash V_{\infty}$ is second countable and we need for a basis of the topology on $\overline{P_{d, \Theta}}$ only countable many open subsets as in a) from Definition A.9. Similarly, since $\Delta$ is second countable we need only countable many open subsets as in b). Finally, for c) since $V_{\infty}$ is countable, we need to argue that each $\xi \in V_{\infty}$ has a countable neighborhood basis. It suffices to vary $U$ over a countable neighborhood basis of $\xi \in \Delta$. Addendum 2.20 implies that for each such $U$ almost all vertices of $\Gamma$ that are incident to $\xi$ (in $P_{d, \Theta}$ ) are contained in $U$. Using Lemma A.4 it follows that all but finitely many of the simplices of $P_{d, \Theta}$ that contain $\xi$ are contained in $P_{d, \Theta}(U)$. Therefore, for each $U$ it suffices to vary $W$ over countable collection of neighborhoods of $\xi \in P_{d, \Theta}$.

b) Let $\xi \in \Delta_{+}, v \in V_{\infty}$. Using Addendum 2.20 we find open neighborhoods $U$ of $\xi$ in $\Delta_{+}$and $W$ of $v$ in $P_{d, \Theta}$ such that $P_{d, \Theta}(U) \cap W=\emptyset$. Combining this observation with the fact that $\Delta_{+}$and $P_{d, \Theta}$ are Hausdorff we see that $\overline{P_{d, \Theta}}$ is also Hausdorff.

Since the topology on $\overline{P_{d, \Theta}}$ has a countable basis it suffices to prove sequential compactness. Let $\left(x_{n}\right)_{n \in \mathbb{N}}$ be a sequence in $\overline{P_{d, \Theta}}$. We will produce a convergent subsequence. If $x_{n} \in \partial \Gamma$ for infinitely many $n$, then we can use the compactness of $\Delta$. Therefore we assume that $x_{n} \in P_{d, \Theta}$ for all $n$. Since $P_{d, \Theta}$ is finite dimensional we find $k$ and vertices $v_{0, n}, \ldots, v_{k, n}$ such that for each $n$ the point $x_{n}$ belongs to the simplex spanned by $v_{0, n}, \ldots, v_{k, n}$. Since $\Delta_{+}$is compact we can assume that $\xi_{j}:=\lim _{n} v_{j, n}$ exists in $\Delta_{+}$for each $j$. We now apply Lemma 2.19] and Addendum 2.20 to the $v_{0, n}, \ldots, v_{k, n}$ as sequences in $n$. It follows that either the 
$\xi_{j}=: \xi$ all coincide or that we find a subsequence $I \subseteq \mathbb{N}$ such that for each $j$, $v_{j, n}=: w_{j}$ is constant in $n \in I$. In the first case $\lim _{n \in \mathbb{N}} x_{n}=\xi$. In the second case we find a subsequence of $x_{n}$ that converges to a point on the simplex spanned by the $w_{j}$.

Since $\overline{P_{d, \Theta}}$ is compact and has a countable basis for the topology it is metrizable.

c) This follows from Theorem A.1 and Lemma A.4 and the fact that the finite union of finite dimensional spaces is finite dimensional [25, p.28].

d) If $\xi \in \overline{P_{d, \Theta}} \backslash \Delta=P_{d, \Theta} \backslash V_{\infty}$, then $\xi$ has arbitrarily small contractible neighborhoods. If $\xi \in \Delta$, then we may assume that $P_{d, \Theta}\left(U_{+}\right) \subseteq U$ for some neighborhood $U_{+}$of $\xi \in \Delta_{+}$. Using Lemma 2.21 we find a smaller neighborhood $U_{+}^{\prime}$ of $\xi$ such that all vertices on geodesics between points of $U_{+}^{\prime}$ belong to $U_{+}$. Proposition A.5 implies that any finite subcomplex of $P_{d, \Theta}\left(U_{+}^{\prime}\right)$ is contractible within $P_{d, \Theta}\left(U_{+}\right)$. If $\xi \in \partial \Gamma$, we can use the interior of $P_{d, \Theta}\left(U_{+}^{\prime}\right) \cup U_{+}^{\prime}$ for $U^{\prime}$. If $\xi \in V_{\infty}$, then we pick in addition an neighborhood $W^{\prime}$ of $\xi$ in $P_{d, \Theta}$ such that $W^{\prime} \subseteq U$. Moreover, since $P_{d, \Theta}\left(U_{+}^{\prime}\right)$ is a subcomplex of $P_{d, \Theta}$, we can assume that $W^{\prime} \cap P_{d, \Theta}\left(U_{+}^{\prime}\right)$ is a deformation retract of $W^{\prime}$. Now we can choose the interior of $P_{d, \Theta}\left(U_{+}^{\prime}\right) \cup U_{+}^{\prime} \cup W^{\prime}$ for $U^{\prime}$

Let $K$ be a simplicial complex and $\mathcal{U}$ be a cover of a space $X$. A map $f: K^{\prime} \rightarrow X$ defined on a subcomplex $K^{\prime}$ of $K$, containing the 0 -skeleton $K^{(0)}$ of $K$, is said to be a partial $\mathcal{U}$-realization if for every simplex $\sigma$ of $K$, there is a member of $\mathcal{U}$ that contains $f\left(K^{\prime} \cap \sigma\right)$. If $K^{\prime}=K$, then $f$ is called a full $\mathcal{U}$-realization.

Lemma A.12. Let $\mathcal{U}$ be an open cover of $\overline{P_{d, \Theta}}$ and $n \in \mathbb{N}$. Then there exists an open cover $\mathcal{U}^{\prime}$ of $\overline{P_{d, \Theta}}$ such that for any finite simplicial complex $K$ of dimension $\leq n$, any partial $i^{-1} \mathcal{U}^{\prime}$-realization of $K$ in $P_{d, \Theta}$ extends to a full $i^{-1} \mathcal{U}$-realization $K \rightarrow P_{d, \Theta}$.

Proof. Using Lemma A.11 d) we find a sequence of successively smaller covers $\mathcal{U}=\mathcal{U}_{n}, \ldots, \mathcal{U}_{0}=\mathcal{U}^{\prime}$ such that for any $U^{\prime} \in \mathcal{U}_{k}$ there is $U \in \mathcal{U}_{k+1}$ such that any map $S^{k} \rightarrow i^{-1}\left(U^{\prime}\right)$ extends to $D^{k+1} \rightarrow i^{-1}(U)$. Inductively, any partial $i^{-1} \mathcal{U}^{\prime}$ realization can then be extended to a $i^{-1} \mathcal{U}$-realization $K \rightarrow P_{d, \Theta}$.

Let $\mathcal{U}$ be a cover of a space $X$. Maps $f, f^{\prime}: Z \rightarrow X$ are said to be $\mathcal{U}$-close if for any $x \in Z$ there is $U \in \mathcal{U}$ containing both $f(x)$ and $f^{\prime}(x)$. The mesh of a cover of a metric space is the supremum of the diameter of its members.

Lemma A.13. For any open cover $\mathcal{W}$ of $\overline{P_{d, \Theta}}$ and any map $f: K \rightarrow \overline{P_{d, \Theta}}$ defined on a finite complex there is a map $f^{\prime}: K \rightarrow P_{d, \Theta}$ such that $f$ and $i \circ f^{\prime}$ are $\mathcal{W}$-close.

Proof. Pick a metric $d_{\bar{P}}$ for $\overline{P_{d, \Theta}}$ and $\varepsilon>0$ such that $3 \varepsilon$ is a Lebesgue number for $\mathcal{W}$. Let $\mathcal{U}$ be a cover of mesh $\leq \varepsilon$. Pick $\mathcal{U}^{\prime}$ as in Lemma A.12 with respect to $\mathcal{U}$ and $n:=\operatorname{dim} K$. Pick $\delta>0$ such that $3 \delta$ is a Lebesgue number for $\mathcal{U}^{\prime}$ and $\delta<\varepsilon$. Now subdivide $K$ until the diameter of the image of each simplex in $\overline{P_{d, \Theta}}$ is at most $\delta$. Since $i\left(P_{d, \Theta}\right)$ is dense in $\overline{P_{d, \Theta}}$ we find $f_{0}^{\prime}: K^{(0)} \rightarrow P_{d, \Theta}$ defined on the 0 -skeleton $K^{(0)}$ of $K$ such that $d_{\bar{P}}\left(i \circ f_{0}^{\prime}(x), f(x)\right) \leq \delta$ for any $x \in K^{(0)}$. It follows that $f_{0}^{\prime}$ is a partial $i^{-1} \mathcal{U}^{\prime}$ realization and therefore extends to a full $i^{-1} \mathcal{U}$-realization $f^{\prime}: K \rightarrow P_{d, \Theta}$. By construction for any $x \in K, d_{\bar{P}}\left(f(x), f^{\prime}(x)\right) \leq 2 \delta+\varepsilon \leq 3 \varepsilon$. Thus $f$ and $i \circ f^{\prime}$ are $\mathcal{W}$-close.

Lemma A.14. Let $f: K \rightarrow \overline{P_{d, \Theta}}$ be a map defined on a finite simplicial complex. Then there exists a homotopy $H: K \times(0,1] \rightarrow P_{d, \Theta}$ such that

$$
(x, t) \mapsto \begin{cases}f(x) & t=0 \\ i(H(x, t)) & t>0\end{cases}
$$


is a continuous homotopy $K \times[0,1] \rightarrow \overline{P_{d, \Theta}}$.

Proof. Let $d_{\bar{P}}$ be again a metric on $\overline{P_{d, \Theta}}$. Let $\mathcal{U}_{l}$ be a sequence of open covers of $\overline{P_{d, \Theta}}$ with mesh $\mathcal{U}_{l} \rightarrow 0$. Let $\mathcal{U}_{l}^{\prime}$ be a cover as in Lemma A.12 with respect to $\mathcal{U}_{l}$ and $n:=\operatorname{dim} K+1$. Let $\lambda_{l}$ be a Lebesgue number for $\mathcal{U}_{l}$. Let $\varepsilon_{l}$ be a sequence such that $\varepsilon_{l}+\varepsilon_{l+1} \leq \lambda_{l}$ for all $l$. Using Lemma A.13 we find maps $f_{l}^{\prime}: K \rightarrow P_{d, \Theta}$ such that $f$ and $i \circ f_{l}^{\prime}$ are $\varepsilon_{l}$-close, i.e., $d_{\bar{P}}\left(f(x), i\left(f_{l}^{\prime}(x)\right)\right)<\varepsilon_{l}$ for all $x \in K$. For $t=1 / l$ set $H(x, 1 / l):=f_{l}^{\prime}(x)$. In order to define $H(x, t)$ for $1 / l+1<t<1 / l$, we can view $f_{l+1}^{\prime} \amalg f_{l}^{\prime}: K \times\{1 / l+1,1 / l\} \rightarrow P_{d, \Theta}$ as a partial $\mathcal{U}_{l}^{\prime}$-realization for an appropriate triangulation of $K \times[1 / l+1,1 / l]$ and use Lemma A.12. Since mesh $\mathcal{U}_{l} \rightarrow 0$ the map $H$ defined this way has the desired continuity property.

Lemma A.15. The space $\overline{P_{d, \Theta}}$ is locally $n$-connected for each $n$, i.e., for each $\xi \in \overline{P_{d, \Theta}}$ and each open neighborhood $U$ of $\xi$, there is a smaller neighborhood $U^{\prime}$ of $\xi$ such that any map $f: S^{k} \rightarrow U^{\prime}$ with $k \leq n$ extends to a map $D^{k+1} \rightarrow U$.

Proof. Pick $U^{\prime}$ as in Lemma A.11 d) Lemma A.14 implies that any map $f: S^{k} \rightarrow$ $U^{\prime}$ is homotopic to a map of the form $i \circ f^{\prime}$ with $f^{\prime}: S^{k} \rightarrow i^{-1}\left(U^{\prime}\right)$. Now $f^{\prime}$ extends to a map $D^{k+1} \rightarrow i^{-1}(U)$ by Lemma A.11 d) and this yields the desired extension of $f$ as well.

Recall that we fixed $d$ and $\Theta$ such that Proposition A.5 applies.

Theorem A.16. The space $\overline{P_{d, \Theta}}$ is a contractible ANR.

Proof. From Lemmas A.11 and A.15 we know that $\overline{P_{d, \Theta}}$ is compact, finite dimensional and locally $n$-connected for any $n$. Since this is a characterization of finite dimensional compact $A N R \mathrm{~s}\left[12\right.$, Thm. 10.3, p.122], $\overline{P_{d, \Theta}}$ is an ANR. Lemma A.14 together with the contractibility of $P_{d, \Theta}$ (Proposition A.5) imply that $\overline{P_{d, \Theta}}$ is weakly contractible. Since finite dimensional $A N R$ s are retracts of simplicial complexes [12, Thm.10.1, p.122] the contractibility of $\overline{P_{d, \Theta}}$ follows.

Remark A.17. Bestvina and Mess 11 proved Theorem A.16 for hyperbolic groups and the argument in this section is closely modeled on their argument. For hyperbolic groups Bestvina and Mess showed moreover that the boundary is a $Z$-set in the analog of $\overline{P_{d, \Theta}}$, but the methods of this section do not give this stronger statement. The reason for this is, that the contractions from Proposition A.5 may not work in $\overline{P_{d, \Theta}} \backslash \Delta=P_{d, \Theta} \backslash V_{\infty}$. For example, if $\Gamma$ is a tree and if we use for $\Theta$ only the trivial angles $(e, e)$, then $P_{d, \Theta}=\Gamma$. Nevertheless, it seems likely that in many cases $\Delta_{+}$is a $Z$-set in $\overline{P_{d, \Theta}}$ for suitable $d, \Theta$.

\section{ApPendix B. EXTENDING OPEN SETS.}

Here we review a convenient procedure to extend open subset to open subsets of an ambient space. Let $\left(X, d_{X}\right)$ be a metric space. Let $X_{0} \subseteq X$ be a subspace. For an open subset $U$ of $X_{0}$ we define $U^{+}:=\left\{x \in X \mid d_{X}(x, U)<d_{X}\left(x, X_{0} \backslash U\right)\right\}$. This construction has the following properties (compare [4, Lem. 4.14]): $U^{+}$is open; $X_{0} \cap U^{+}=U ;(U \cap V)^{+}=U^{+} \cap V^{+}$. We record the following two consequences.

Lemma B.1. For any open subset $U \subseteq X_{0}$, the open subset $U^{+} \subseteq X$ satisfies $U=X_{0} \cap U^{+}$and $\partial_{0} U=X_{0} \cap \partial U^{+}$where $\partial_{0}$ is the boundary in $X_{0}$ and $\partial$ is the boundary in $X$.

Proof. We already know that $U^{+}$is open and satisfies $U=X_{0} \cap U^{+}$. This implies $\partial_{0} U \subseteq X_{0} \cap \partial U \subseteq X_{0} \backslash U$. Let $V$ be the complement of the closure of $U$ in $X_{0}$. Then $V^{+}$is open and $V^{+} \cap U^{+}=\emptyset$. Thus $V^{+} \cap \partial U^{+}=\emptyset$. Thus $X_{0} \cap \partial U^{+} \subseteq$ $X_{0} \backslash(U \cap V)=\partial_{0} U$. 
Suppose now that $G$ acts isometrically on $X$ and that $X_{0} \subseteq X$ is $G$-invariant.

Lemma B.2. Let $\mathcal{F}$ be a family of subgroups of $G$. Let $\mathcal{U}$ be a $G$-invariant collection of $\mathcal{F}$-subsets of $X_{0}$. Then $\mathcal{U}^{+}:=\left\{U^{+} \mid U \in \mathcal{U}\right\}$ is a $G$-invariant collection of $\mathcal{F}$ subsets of $X$ extending the $U \in \mathcal{U}$. The order of $\mathcal{U}$ and $\mathcal{U}^{+}$agree.

Proof. We have $(g U)^{+}=g\left(U^{+}\right)$as the action is isometric. This implies that $\mathcal{U}^{+}$ consists of $\mathcal{F}$-subsets and that $\mathcal{U}^{+}$is $G$-invariant. The equation $(U \cap V)^{+}=U^{+} \cap V^{+}$ implies that the orders of $\mathcal{U}$ and $\mathcal{U}^{+}$agree.

\section{ApPendix C. IDEAL TRIANGLES ARE SLIM}

Lemma C.1. Let $\Gamma$ be a $\delta$-hyperbolic graph, i.e., all finite geodesic triangles are $\delta$-slim. Then all geodesic triangles, including those with one or more corners on the boundary $\partial \Gamma$ are $5 \delta$-slim.

Proof. Let $c, c^{\prime}$ and $c^{\prime \prime}$ be the three sides of a geodesic triangles. Write $\xi, \xi^{\prime}$ and $\xi^{\prime \prime}$ for the three corners of the triangle, i.e., $c$ is geodesic between $\xi^{\prime}$ and $\xi^{\prime \prime}, c^{\prime}$ is a geodesic between $\xi$ and $\xi^{\prime \prime}$, and $c^{\prime \prime}$ is a geodesic between $\xi$ and $\xi^{\prime}$. We pick vertices $y \in c, y^{\prime} \in c^{\prime}$ and $y^{\prime \prime} \in c^{\prime \prime}$, We write $c_{\xi^{\prime}}$ for the restriction of $c$ to a geodesic from $y$ to $\xi^{\prime}$. Similarly, we define $c_{\xi^{\prime \prime}}, c_{\xi}^{\prime}, c_{\xi^{\prime \prime}}^{\prime \prime}, c_{\xi}^{\prime \prime}$ and $c_{\xi^{\prime}}^{\prime \prime}$. These six geodesics are asymptotic in three pairs. Thus we find $A>0$ such that the Hausdorff distances between $c_{\xi}^{\prime}$ and $c_{\xi}^{\prime \prime}$, and between $c_{\xi^{\prime}}$ and $c_{\xi^{\prime}}^{\prime \prime}$, and between $c_{\xi^{\prime \prime}}$ and $c_{\xi^{\prime \prime}}^{\prime \prime}$ are all three $\leq A$.

Let now $v$ be a vertex on $c$. We pick a geodesic $a$ of length at most $A$ that starts on $c^{\prime}$ and ends on $c^{\prime \prime}$. If $\xi \in \partial \Gamma$, then we can assume in addition that $d_{\Gamma}(v, a) \geq 100(A+\delta)$. If $\xi$ is a vertex of $\Gamma$ then we assume that $a$ is the constant geodesic at $\xi$. We pick a vertex $x$ on $a$. Similarly, we pick geodesics $a^{\prime}$ and $a^{\prime \prime}$ with vertices $x^{\prime} \in a^{\prime}$ and $x^{\prime \prime} \in a^{\prime \prime}$. We can assume that along $c$ the vertex $v$ is between $a^{\prime} \cap c$ and $a^{\prime \prime} \cap c$; if not then move $a^{\prime}$ towards $\xi^{\prime}$ and $a^{\prime \prime}$ towards $\xi^{\prime \prime}$. We also pick geodesics $b$ from $x^{\prime}$ to $x^{\prime \prime}, b^{\prime}$ from $x$ to $x^{\prime \prime}$, and $b^{\prime \prime}$ from $x$ to $x^{\prime}$.

Consider now the geodesic 4-gon whose four sides are $b$, and restrictions of $a^{\prime}, c$ and $a^{\prime \prime}$. Since $a^{\prime}$ and $a^{\prime \prime}$ are either constant or far from $v$ we can apply hyperbolicity and find a vertex $w_{1} \in b$ with $d_{\Gamma}\left(v, w_{1}\right) \leq 2 \delta$. Next we use the geodesic triangle with sides $b, b^{\prime}$ and $b^{\prime \prime}$. By hyperbolicity there is $w_{2} \in b^{\prime} \cup b^{\prime \prime}$ with $d_{\Gamma}\left(w_{1}, w_{2}\right) \leq \delta$. Without of loss of generality we assume $w_{2} \in b^{\prime}$. Now we consider the geodesic 4-gon whose four sides are $b^{\prime}$ and restrictions of $a, c^{\prime}$ and $a^{\prime \prime}$. Since $a$ and $a^{\prime \prime}$ are either constant or far from $v$ (and then also far from $w_{2}$ ) we can apply hyperbolicity and find a vertex $v^{\prime} \in c^{\prime}$ with $d_{\Gamma}\left(w_{2}, v^{\prime}\right) \leq 2 \delta$. Altogether, $d_{\Gamma}\left(v, v^{\prime}\right) \leq 5 \delta$ and $v^{\prime} \in c^{\prime} \cup c^{\prime \prime}$.

\section{REFERENCES}

[1] Y. Antolín, R. Coulon, and G. Gandini. Farrell-Jones via Dehn fillings. Preprint, arXiv:1510.08113v1, 2015.

[2] A. Bartels, F. T. Farrell, and W. Lück. The Farrell-Jones conjecture for cocompact lattices in virtually connected Lie groups. J. Amer. Math. Soc., 27(2):339-388, 2014.

[3] A. Bartels and W. Lück. The Borel conjecture for hyperbolic and CAT(0)-groups. Ann. of Math. (2), 175:631-689, 2012.

[4] A. Bartels and W. Lück. Geodesic flow for CAT(0)-groups. Geom. Topol., 16:1345-1391, 2012.

[5] A. Bartels, W. Lück, and H. Reich. Equivariant covers for hyperbolic groups. Geom. Topol., 12(3):1799-1882, 2008.

[6] A. Bartels, W. Lück, and H. Reich. The $K$-theoretic Farrell-Jones conjecture for hyperbolic groups. Invent. Math., 172(1):29-70, 2008.

[7] A. Bartels, W. Lück, and H. Reich. On the Farrell-Jones Conjecture and its applications. Journal of Topology, 1:57-86, 2008. 
[8] A. Bartels, W. Lück, H. Reich, and H. Rüping. K- and L-theory of group rings over $G L_{n}(\mathbf{Z})$. Publ. Math. Inst. Hautes Études Sci., 119:97-125, 2014.

[9] A. Bartels and H. Reich. Coefficients for the Farrell-Jones Conjecture. Adv. Math., 209(1):337-362, 2007.

[10] H. Bass. The degree of polynomial growth of finitely generated nilpotent groups. Proc. London Math. Soc. (3), 25:603-614, 1972.

[11] M. Bestvina and G. Mess. The boundary of negatively curved groups. J. Amer. Math. Soc., 4(3):469-481, 1991.

[12] K. Borsuk. Theory of retracts. Monografie Matematyczne, Tom 44. Państwowe Wydawnictwo Naukowe, Warsaw, 1967.

[13] B. H. Bowditch. Relatively hyperbolic groups. Internat. J. Algebra Comput., 22(3):1250016, $66,2012$.

[14] M. R. Bridson and A. Haefliger. Metric spaces of non-positive curvature. Springer-Verlag, Berlin, 1999. Die Grundlehren der mathematischen Wissenschaften, Band 319.

[15] F. Dahmani. Classifying spaces and boundaries for relatively hyperbolic groups. Proc. London Math. Soc. (3), 86(3):666-684, 2003.

[16] J. F. Davis, F. Quinn, and H. Reich. Algebraic $K$-theory over the infinite dihedral group: a controlled topology approach. J. Topol., 4(3):505-528, 2011.

[17] T. Elsner. Systolic groups with isolated flats. Preprint, 2008.

[18] B. Farb. Relatively hyperbolic groups. Geom. Funct. Anal., 8(5):810-840, 1998.

[19] F. T. Farrell and L. E. Jones. K-theory and dynamics. I. Ann. of Math. (2), 124(3):531-569, 1986.

[20] F. T. Farrell and L. E. Jones. Isomorphism conjectures in algebraic K-theory. J. Amer. Math. Soc., 6(2):249-297, 1993.

[21] F. T. Farrell and S. K. Roushon. The Whitehead groups of braid groups vanish. Internat. Math. Res. Notices, 10:515-526, 2000.

[22] M. Gromov. Hyperbolic groups. In Essays in group theory, pages 75-263. Springer-Verlag, New York, 1987.

[23] M. Gromov. Asymptotic invariants of infinite groups. In Geometric group theory, Vol. 2 (Sussex, 1991), pages 1-295. Cambridge Univ. Press, Cambridge, 1993.

[24] D. Groves and J. F. Manning. Dehn filling in relatively hyperbolic groups. Israel J. Math., 168:317-429, 2008.

[25] W. Hurewicz and H. Wallman. Dimension Theory. Princeton Mathematical Series, v. 4. Princeton University Press, Princeton, N. J., 1941.

[26] D. Kasprowski and H. Rüping. Long and thin covers for cocompact flow spaces. Preprint, arXiv:1502.05001 2015.

[27] W. Lück. $K$ - and $L$-theory of group rings. In Proceedings of the International Congress of Mathematicians. Volume II, pages 1071-1098. Hindustan Book Agency, New Delhi, 2010.

[28] W. Lück and H. Reich. The Baum-Connes and the Farrell-Jones conjectures in $K$ - and $L$ theory. In Handbook of K-theory. Vol. 1, 2, pages 703-842. Springer, Berlin, 2005.

[29] I. Mineyev. Flows and joins of metric spaces. Geom. Topol., 9:403-482 (electronic), 2005.

[30] I. Mineyev and A. Yaman. Relative hyperbolicity and bounded cohomology. Preprint, http://www.math.uiuc.edu/ mineyev/math/art/rel-hyp.pdf

[31] A. Mole. Extending a metric on a simplicial complex. Preprint, arXiv:1309.0981 [math.AT], 2013.

[32] A. Mole and H. Rüping. Equivariant refinements. Preprint, arXiv:1308.2799, 2013.

[33] D. Osin. Asymptotic dimension of relatively hyperbolic groups. Int. Math. Res. Not., (35):2143-2161, 2005.

[34] N. Ozawa. Amenable actions and applications. In International Congress of Mathematicians. Vol. II, pages 1563-1580. Eur. Math. Soc., Zürich, 2006.

[35] N. Ozawa. Boundary amenability of relatively hyperbolic groups. Topology Appl., 153(14):2624-2630, 2006.

[36] A. R. Pears. Dimension theory of general spaces. Cambridge University Press, Cambridge, England-New York-Melbourne, 1975.

[37] F. Quinn. Algebraic K-theory over virtually abelian groups. J. Pure Appl. Algebra, 216(1):170-183, 2012.

[38] R. Sauer. Amenable covers, volume and $L^{2}$-Betti numbers of aspherical manifolds. J. Reine Angew. Math., 636:47-92, 2009.

[39] G. Szabó, J. Wu, and J. Zacharias. Rohklin dimension for actions of residually finite groups. Preprint, arXiv:1408.6096 2014.

[40] A. Szczepański. Relatively hyperbolic groups. Michigan Math. J., 45(3):611-618, 1998.

[41] C. Wegner. The $K$-theoretic Farrell-Jones conjecture for CAT(0)-groups. Proc. Amer. Math. Soc., 140(3):779-793, 2012. 
E-mail address: a.bartels@wwu.de

Westfälische Wilhelms-Universität Münster, Mathematisches Institut, Einsteinstr. 62 , D-48149 Münster, Germany 